\title{
New approach for the analysis of damped vibrations of fractional oscillators
}

\author{
Yuriy A. Rossikhin and Marina V. Shitikova* \\ Voronezh State University of Architecture and Civil Engineering, Voronezh 394006, Russia
}

Received 29 February 2008

Revised 27 May 2008

\begin{abstract}
The dynamic behavior of linear and nonlinear mechanical oscillators with constitutive equations involving fractional derivatives defined as a fractional power of the operator of conventional time-derivative is considered. Such a definition of the fractional derivative enables one to analyse approximately vibratory regimes of the oscillator without considering the drift of its position of equilibrium. The assumption of small fractional derivative terms allows one to use the method of multiple time scales whereby a comparative analysis of the solutions obtained for different orders of low-level fractional derivatives and nonlinear elastic terms is possible to be carried out. The interrelationship of the fractional parameter (order of the fractional operator) and nonlinearity manifests itself in full measure when orders of the small fractional derivative term and of the cubic nonlinearity entering in the oscillator's constitutive equation coincide.
\end{abstract}

Keywords: Fractional oscillator, linear and nonlinear vibrations, method of multiple time scales

\section{Introduction}

The study of dynamical behavior of the fractional order systems has started to attract increasing attention in the last few decades, since it has been found that in interdisciplinary fields many systems can be described by equations that consist of derivatives of fractional order [1-7]. Considerable recent attention has been focused on nonlinear vibrations of so called 'fractional oscillators'. Fractional oscillator is a notion that appears in the literature, principally in the field of physics [8,9], for an oscillator with the second derivative replaced by a fractional one of order less than two, in so doing Riemann-Liouville, Gr ünvald-Letnikov, Caputo or Riesz definitions for the general fractional differintegral have been utilized.

Thus, dynamics of the fractional order Van der Pol oscillator

$$
\begin{aligned}
& D^{\alpha+1} y+y+a\left(y^{2}-1\right) D^{\alpha} y=f(t) \\
& \ddot{y}+y+a\left(y^{2}-1\right) D^{\alpha} y=f(t)
\end{aligned}
$$

has been studied for Eq. (1a) in [10] using the approximate frequency method, where $y$ is the dependent variable, $a>0$ is a control parameter that reflects the degree of nonlinearity of the system, $0<\alpha<1$ is the order of the fractional derivative $D^{\alpha}$, and $f(t)$ is the exciting force. Equation (1b) at $0<\alpha<2$ has been utilized in [11].

The fractional order Duffing-like systems with negative linear stiffness

$$
D^{\alpha} y-y+a y^{3}=f(t),
$$

\footnotetext{
${ }^{*}$ Corresponding author. E-mail: shitikova@ vmail.ru.
} 


$$
\begin{aligned}
& D^{2-\alpha} y-y+a y^{3}=f(t), \\
& D^{\alpha+1} y-y+y^{3}+\delta D^{\alpha} y=f(t),
\end{aligned}
$$

where $a$ and $\delta$ are some positive parameters, have been analyzed for $1<\alpha<2$ using Eq. (2a) in [9], for very small magnitudes of $\alpha$ using Eq. (2b) in [12], and for $0<\alpha<1$ using Eq. (2c) in [2].

An extensive literature on other fractional order systems describing different physical phenomena can be found in $[7,12]$.

But for mechanical and civil engineering applications the introduction of a fractional derivative into inertial terms of equations of motion is neither attractive nor justified. In engineering practice, fractional derivatives are useful for describing viscoelastic features of advanced materials or dissipative forces in structural dynamics $[1,6]$. The exact solutions for linear fractional oscillators based on different fractional calculus constitutive equations can be found in $[13,14]$, where the solution is presented via Laplace transform technique.

Fractional derivatives are among other things useful in describing the frequency dependent damping behavior of nonlinear structural systems as well [15-20]. Since the methods of integral transformations are unusable in nonlinear problems, so different methods of perturbation technique or numerical methods are overwhelmingly used for investigating vibrations of such nonlinear structures.

Recently dynamics of the fractionally damped Duffing oscillator has been examined by several authors [17,21-26]. Thus, the Duffing-like oscillator with positive linear stiffness and damping defined by a fractional derivative

$$
m \ddot{x}(t)+c D_{q} x(t)+k x(t)+\varepsilon x(t)^{3}=f \cos (w t),
$$

where $x, m, c, k$, and $\varepsilon$ are respectively the oscillator's displacement, mass, damping coefficient, linear stiffness, and small parameter of nonlinear stiffness, has been studied in [17] by energy constrained Lindstedt-Poincar é perturbation procedure employing a diophantine version of the fractional operator $D_{q}$ powers. The influence of fractional damping on the frequency amplitude response has been examined.

The case of $f=0$ and half-order Riemann-Liouville fractional derivative in Eq. (3) was analysed in [21] applying variational iteration method allowing one to obtain approximate analytical solution.

The occurrence and the nature of chaotic motions described by Eq. (3) with negative coefficient $k$ have been studied using different numerical methods in [22], in so doing Caputo type fractional derivative has been utilized. Galerkin projection method and finite element method were adopted in [25] for solving equation similar to Eq. (3) but with negative linear stiffness and the dissipative force modeled via the Riemann-Liouville fractional derivative of the half-order, while the possibilities of using other magnitudes of the fractional parameter were also discussed.

The method of averaging was applied in [26] for investigating the following equations with different type of small damping including fractional derivative terms and delayed terms

$$
\begin{aligned}
& \ddot{x}(t)+\varepsilon D^{1 / 2} x(t)+x(t)=\delta(t), \\
& \ddot{x}(t)+x(t)^{3}=-\varepsilon D^{1 / 2} x(t), \\
& \ddot{x}(t)+x(t)^{3}=-\varepsilon\left[\alpha \dot{x}(t)+\beta D^{1 / 2} x(t)+\gamma \dot{x}(t-1)\right], \\
& \ddot{x}(t)+x(t)|x(t)|=-\varepsilon\left[\alpha \dot{x}(t)+\beta D^{1 / 2} x(t)+\gamma \dot{x}(t-1)\right],
\end{aligned}
$$

where $D^{1 / 2}$ is the Riemann-Liouville half-order derivative.

The fractional oscillator with nonlinear elasticity incorporated in the form of the third and fifth order polynomial stiffness terms was studied in [27].

Although for the mechanical engineer or civil engineer, a term fractionally damped structure should mean application of fractional calculus representations for describing damping forces in governing equations of motion, some authors use the notion of fractional damping for denoting the dissipative force which is introduced as a 
fractional-power law of the velocity [28-33]. In other words, nonlinear equations with fractional-power nonlinearities are considered.

For example, the following second order differential equation

$$
\ddot{x}+\varepsilon(\dot{x})^{1 / 3}+x=0, \quad \varepsilon>0,
$$

has been studied in [28] using Krylov-Bogoliubov method.

Dynamics of a single-degree-of-freedom system with nonlinear viscous damping via the fractional-power law of the velocity

$$
m \ddot{x}(t)+c \operatorname{sign}[\dot{x}(t)]|\dot{x}(t)|^{\alpha}+k x(t)=f(t),
$$

where sign is a signum function, and $\alpha<1$ is damping law exponent, has been examined in [31] by direct numerical integration of Eq. (5b).

Duffing-like oscillator obtained from Eq. (5b) by adding the Duffing term

$$
m \ddot{x}(t)+c \operatorname{sign}[\dot{x}(t)]|\dot{x}(t)|^{\alpha}+k x(t)+\varepsilon x(t)^{3}=f(t),
$$

was examined in [32] by the analytical Melnikov method and numerical simulations considering a negative linear term.

Note that sometimes confusion in understanding the notion of fractional damping can be made by incorrect treatment of the representation of fractional derivative in the form of $(d / d t)^{\alpha}$ [34]. However,

$$
\left(\frac{d}{d t}\right)^{\alpha} x(t) \neq\left(\frac{d}{d t} x(t)\right)^{\alpha} .
$$

In the present paper, contrary to other articles devoted to fractional oscillators, an engineering approach is implemented to the analysis of free vibrations of mechanical oscillators, constitutive equations of which involve fractional derivatives. This approach lies in the fact that it takes no account of the drift of the equilibrium position of the oscillator, which, as experiments show, can be ignored. Neglect of the equilibrium position drift resides already in the definition of the fractional derivative, as such, since the fractional derivative in this paper is determined as a fractional power of the operator of conventional time-derivative $d / d t$, while this operator, as it has been shown in [34], is equivalent to the Riemann-Liouville fractional derivative in time $t$, i.e.,

$$
D_{+}^{\gamma} x(t)=\frac{d}{d t} \int_{-\infty}^{t} \frac{x\left(t-t^{\prime}\right) d t^{\prime}}{\Gamma(1-\gamma) t^{\gamma}}
$$

that is (see formula (5.82) in [34])

$$
\left(\frac{d}{d t}\right)^{\gamma} x(t)=D_{+}^{\gamma} x(t) .
$$

Since the low limit of the integral is equal to $-\infty$, then this fact allows one to use the formula [34]

$$
D_{+}^{\gamma} e^{i \omega t}=(i \omega)^{\gamma} e^{i \omega t},
$$

which becomes invalid in the case of equality to zero of the low limit. For this case there exists another formula (Appendix A)

$$
D_{0+}^{\gamma} e^{i \omega t}=(i \omega)^{\gamma} e^{i \omega t}+\frac{\sin \pi \gamma}{\pi} \int_{0}^{\infty} \frac{u^{\gamma} e^{-u t} d u}{u+i \omega},
$$

where

$$
D_{0+}^{\gamma} e^{i \omega t}=\frac{d}{d t} \int_{0}^{t} \frac{e^{i \omega\left(t-t^{\prime}\right)} d t^{\prime}}{\Gamma(1-\gamma) t^{\prime \gamma}}
$$

which goes over in Eq. (9) when $t \rightarrow+\infty$.

In all examples considered below we will ignore the drift of the equilibrium position of the fractional oscillator, and, thus, our goal is to obtain the approximate analytical solutions using Eq. (9) for calculating the fractional derivative of the exponential function. 
If one uses the exact formula for fractional differentiation of the exponent Eq. (10), then the integral entering in Eq. (10) determines that part of the solution which describes the drift of the position of oscillator's equilibrium [1]. However, this integral decays rapidly with time, and in many cases (see Appendix) it can be neglected as compared with the first term in Eq. $(10)[15,16,18]$. Reference to Eq. (10) shows that if $\gamma$ is rather small, while the frequency $\omega$ lies within the range of engineering interest, then the integral in the right-hand side of Eq. (10) can be ignored, what allows one to use Eq. (9).

Thus, calculations of the magnitude of the fractional parameters carried out on the basis of the experimental data [35] show that this value for suspension bridges has the order of $0.05-0.1[16,36]$. The magnitude of $\gamma=0.118$ was reported in [37] when studying the experimental response of a deformable single-link mechanism, which was realized by means of a brass bar fixed onto a vertical shaft. The fractional parameters $\gamma_{1}=0.1991$ and $\gamma_{2}=0.2499$ were identified in [38] while carrying out experimental measurements of a cantilever made of Delrin ${ }^{\mathrm{TM}}$. The magnitude of $\gamma=0.28$ was obtained in [39] in the beam impact experiment. A series of experiments measuring the frequency responses of viscoelastic rods of materials like Teflon, PolyAmid, PolyUrethan, PolyVinylChlorid, and PolyEthylen was reported in $[40,41]$, where it was found that the fractional parameter lies in the range of $0.086-0.11$. During flexible polyurethan foam modeling via a nonlinear fractional oscillator in [27], viscoelastic parameters for automotive seating applications were identified with the fractional parameter equal to 0.019 .

The necessity of studying fractional oscillators is motivated by two reasons: first, engineers often use 1DOF models as a first approximation or as a benchmark before preceding to more intricate models or multi-degree-of-freedom structural systems (for example, as the simplest model of a vibration-isolation system [42-49]), and second, the study of vibrations of more complex structures can be reduced to vibrations of a set of fractional oscillators [50-53].

\section{Free damped vibrations of a linear oscillator in a medium with small viscousity}

Let us consider free damped vibrations of a mechanical oscillator, the equation of motion of which has the form

$$
\ddot{x}+\omega_{0}^{2} x=-2 \varepsilon æ\left(\frac{d}{d t}\right)^{\gamma} x,
$$

where $\omega_{0}^{2}=E_{0} m^{-1}, E_{0}$ is the elastic modulus, $m$ is the mass of the oscillator, $x$ is its deviation from the position of equilibrium, $t$ is the time, an overdot denotes the time-derivative, $\gamma(0<\gamma \leqslant 1)$ is the fractional parameter, $\varepsilon \ngtr$ is the coefficient of viscosity per unit mass, and $\varepsilon$ is a small parameter.

The desired function $x(t)$ can be represented in terms of a series [54]

$$
x(t)=\varepsilon x_{1}\left(T_{0}, T_{1}, \ldots\right)+\varepsilon^{2} x_{2}\left(T_{0}, T_{1}, \ldots\right)+\ldots,
$$

where $T_{n}=\varepsilon^{n} t$.

Then the first, the second and fractional derivatives are defined, respectively, as follows

$$
\begin{aligned}
& \frac{d}{d t}=D_{0}+\varepsilon D_{1}+\varepsilon^{2} D_{2}+\ldots, \\
& \frac{d^{2}}{d t^{2}}=D_{0}^{2}+2 \varepsilon D_{0} D_{1}+\varepsilon^{2}\left(D_{1}^{2}+2 D_{0} D_{2}\right)+\ldots, \\
& \left(\frac{d}{d t}\right)^{\gamma}=\left(D_{0}+\varepsilon D_{1}+\varepsilon^{2} D_{2}+\ldots\right)^{\gamma} \\
& \quad=D_{+}^{\gamma}+\varepsilon \gamma D_{+}^{\gamma-1} D_{1}+\frac{1}{2} \varepsilon^{2} \gamma\left[(\gamma-1) D_{+}^{\gamma-2} D_{1}^{2}+2 D_{+}^{\gamma-1} D_{2}\right]+\ldots,
\end{aligned}
$$

where $D_{n}=\partial / \partial T_{n}$, and $D_{+}^{\gamma}, D_{+}^{\gamma-1}, D_{+}^{\gamma-2}, \ldots$ are the Riemann-Liouville fractional derivatives in time $t$, i.e.,

$$
D_{+}^{\gamma-n} x=\frac{d}{d t} \int_{-\infty}^{t} \frac{x\left(t-t^{\prime}\right) d t^{\prime}}{\Gamma(1-\gamma+n) t^{\prime \gamma-n}} \quad(n=0,1,2 \ldots)
$$


Substituting relationships (12) and (13) in Eq. (11), equating the coefficients at equal powers of $\varepsilon$, and limiting ourselves by terms of $\varepsilon^{4}$, we are led to a set of recurrent equations

$$
\begin{aligned}
D_{0}^{2} x_{1}+\omega_{0}^{2} x_{1}= & 0 \\
D_{0}^{2} x_{2}+\omega_{0}^{2} x_{2}= & -2 æ D_{+}^{\gamma} x_{1}-2 D_{0} D_{1} x_{1}, \\
D_{0}^{2} x_{3}+\omega_{0}^{2} x_{3}= & -2 æ D_{+}^{\gamma} x_{2}-2 D_{0} D_{1} x_{2}-2 æ \gamma D_{+}^{\gamma-1} D_{1} x_{1}-\left(D_{1}^{2}+2 D_{0} D_{2}\right) x_{1}, \\
D_{0}^{2} x_{4}+\omega_{0}^{2} x_{4}= & -2 æ D_{+}^{\gamma} x_{3}-2 D_{0} D_{1} x_{3}-2 æ \gamma D_{+}^{\gamma-1} D_{1} x_{2}-\left(D_{1}^{2}+2 D_{0} D_{2}\right) x_{2} \\
& -æ \gamma\left[(\gamma-1) D_{+}^{\gamma-2} D_{1}^{2}+2 D_{+}^{\gamma-1} D_{2}\right] x_{1}-2\left(D_{0} D_{3}+D_{1} D_{2}\right) x_{1} .
\end{aligned}
$$

The general solution of Eq. (14a) has the form

$$
x_{1}=A_{1}\left(T_{1}, T_{2}, T_{3}\right) e^{i \omega_{0} T_{0}}+\bar{A}_{1}\left(T_{1}, T_{2}, T_{3}\right) e^{-i \omega_{0} T_{0}},
$$

where $A_{1}$ and $\bar{A}_{1}$ are yet unknown complex conjugate functions.

Substituting expression Eq. (15) in the right-hand side of Eq. (14b) and considering Eq. (9), we obtain

$$
D_{0}^{2} x_{2}+\omega_{0}^{2} x_{2}=-2 i \omega_{0}\left[\left(i \omega_{0}\right)^{\gamma-1} æ A_{1}+D_{1} A_{1}\right] e^{i \omega_{0} T_{0}}+2 i \omega_{0}\left[\left(-i \omega_{0}\right)^{\gamma-1} æ \bar{A}_{1}+D_{1} \bar{A}_{1}\right] e^{-i \omega_{0} T_{0}} .
$$

Applying the known procedure for eliminating secular terms, from Eq. (16) we find

$$
D_{1} A_{1}+\left(i \omega_{0}\right)^{\gamma-1} æ A_{1}=0,
$$

whence it follows that

$$
A_{1}\left(T_{1}, T_{2}, T_{3}\right)=a_{1}\left(T_{2}, T_{3}\right) \exp \left[-\left(i \omega_{0}\right)^{\gamma-1} æ T_{1}\right],
$$

where $a_{1}\left(T_{2}, T_{3}\right)$ is yet unknown function.

Substitution of Eq. (17) in the right-hand side of Eq. (16) vanishes it. Then the solution of Eq. (16) takes the form

$$
x_{2}=A_{2}\left(T_{1}, T_{2}, T_{3}\right) e^{i \omega_{0} T_{0}}+\bar{A}_{2}\left(T_{1}, T_{2}, T_{3}\right) e^{-i \omega_{0} T_{0}},
$$

where $A_{2}$ and $\bar{A}_{2}$ are yet unknown complex conjugate functions.

Substituting Eqs (15) and (18) in the right-hand side of Eq. (14c) yields

$$
\begin{aligned}
D_{0}^{2} x_{3}+\omega_{0}^{2} x_{3}= & Q_{1}\left(T_{1}, T_{2}, T_{3}\right) e^{i \omega_{0} T_{0}}+\bar{Q}_{1}\left(T_{1}, T_{2}, T_{3}\right) e^{-i \omega_{0} T_{0}}, \\
Q_{1}\left(T_{1}, T_{2}, T_{3}\right)= & -2 i \omega_{0}\left[\left(i \omega_{0}\right)^{\gamma-1} æ A_{2}+D_{1} A_{2}\right] \\
& +\left[æ^{2}\left(i \omega_{0}\right)^{2(\gamma-1)}(2 \gamma-1) a_{1}-2 i \omega_{0} D_{2} a_{1}\right] \exp \left[-\left(i \omega_{0}\right)^{\gamma-1} æ T_{1}\right] .
\end{aligned}
$$

The terms in the right part of Eq. (19a) produce circular terms. In order to eliminate them, it is necessary to vanish to zero the values in Eq. (19b), i.e.,

$$
D_{1} A_{2}+\left(i \omega_{0}\right)^{\gamma-1} æ A_{2}=\left[\frac{1}{2} æ^{2}\left(i \omega_{0}\right)^{2 \gamma-3}(2 \gamma-1) a_{1}-D_{2} a_{1}\right] \exp \left[-\left(i \omega_{0}\right)^{\gamma-1} æ T_{1}\right] .
$$

The general solution of Eq. (20) has the form

$$
A_{2}=\left\{a_{2}\left(T_{2}, T_{3}\right)+\left[\frac{1}{2} æ^{2}\left(i \omega_{0}\right)^{2 \gamma-3}(2 \gamma-1) a_{1}-D_{2} a_{1}\right] T_{1}\right\} \exp \left[-\left(i \omega_{0}\right)^{\gamma-1} æ T_{1}\right],
$$

where $a_{2}\left(T_{2}, T_{3}\right)$ is yet unknown function.

To eliminate circular terms in Eq. (21), it is a need to equate to zero the expression in square brackets. i.e., 


$$
D_{2} a_{1}-\frac{1}{2} æ^{2}\left(i \omega_{0}\right)^{2 \gamma-3}(2 \gamma-1) a_{1}=0,
$$

whence it follows that

$$
a_{1}=a_{11}\left(T_{3}\right) \exp \left[\frac{1}{2} \Re^{2}\left(i \omega_{0}\right)^{2 \gamma-3}(2 \gamma-1) T_{2}\right],
$$

where $a_{11}\left(T_{3}\right)$ is yet unknown function.

With due account for Eq. (22), expression Eq. (21) takes the form

$$
A_{2}=a_{2}\left(T_{2}, T_{3}\right) \exp \left[-\left(i \omega_{0}\right)^{\gamma-1} æ T_{1}\right],
$$

while the solution of Eq. (19a) is

$$
x_{3}=A_{3}\left(T_{1}, T_{2}, T_{3}\right) e^{i \omega_{0} T_{0}}+\bar{A}_{3}\left(T_{1}, T_{2}, T_{3}\right) e^{-i \omega_{0} T_{0}},
$$

where $A_{3}$ and $\bar{A}_{3}$ are yet unknown complex conjugate functions.

Substituting all functions in the right-hand side of Eq. (14d) and eliminating circular terms in the similar fashion as it has been done above, we can obtain the following:

$$
\begin{aligned}
& a_{2}\left(T_{2}, T_{3}\right)=a_{22}\left(T_{3}\right) \exp \left[\frac{1}{2} \Re^{2}\left(i \omega_{0}\right)^{2 \gamma-3}(2 \gamma-1) T_{2}\right], \\
& a_{11}\left(T_{3}\right)=a_{111} \exp \left[-\frac{1}{2} \Re^{3}\left(i \omega_{0}\right)^{3 \gamma-5}(1-\gamma)(1-3 \gamma) T_{3}\right],
\end{aligned}
$$

where $a_{22}\left(T_{3}\right)$ is yet unknown function, and $a_{111}$ is an unknown constant to be determined from the initial conditions.

Considering relationships Eqs (15), (16), (22), (18), (23), (25), and (26) we find from Eq. (12)

$$
\begin{aligned}
x= & \left\{\varepsilon a_{111} \exp \left[-\frac{1}{2} \mathfrak{x}^{3}\left(i \omega_{0}\right)^{3 \gamma-5}(1-\gamma)(1-3 \gamma) T_{3}\right]+\varepsilon^{2} a_{22}\left(T_{3}\right)\right\} \\
& \times \exp \left(i \omega_{0} T_{0}\right) \exp \left[-\left(i \omega_{0}\right)^{\gamma-1} \rightsquigarrow T_{1}\right] \exp \left[\frac{1}{2} \Re^{2}\left(i \omega_{0}\right)^{2 \gamma-3}(2 \gamma-1) T_{2}\right]+c c
\end{aligned}
$$

where the symbol $c c$ denotes the expression to be complex conjugate to the preceding one.

If we carry out the expansion upto the fifth power of $\varepsilon$, then the function $a_{22}\left(T_{3}\right)$ can be obtained in the following form:

$$
a_{22}\left(T_{3}\right)=a_{222} \exp \left[-\frac{1}{2} æ^{3}\left(i \omega_{0}\right)^{3 \gamma-5}(1-\gamma)(1-3 \gamma) T_{3}\right],
$$

where $a_{222}$ is unknown constant to be determined from the initial conditions.

Substituting Eq. (28) in Eq. (27) and putting

$$
\varepsilon a_{111}+\varepsilon^{2} a_{222}=\frac{1}{2} \varepsilon a_{0} \exp \left(i \varphi_{0}\right),
$$

where $a_{0}$ and $\varphi_{0}$ are new unknown constants, and substituting $T_{n}$ with $\varepsilon^{n} t$, we find

$$
\begin{aligned}
x^{\text {lin.os. }}= & \varepsilon a_{0} \exp \left\{-\varepsilon æ t\left(\omega_{0}\right)^{\gamma-1}\left[\sin \left(\frac{1}{2} \pi \gamma\right)+\frac{1}{2} \varepsilon æ(2 \gamma-1)\left(\omega_{0}\right)^{\gamma-2} \sin \pi \gamma\right.\right. \\
& \left.\left.+\frac{1}{2} \varepsilon^{2} æ^{2}(1-\gamma)(1-3 \gamma)\left(\omega_{0}\right)^{2(\gamma-2)} \sin \left(\frac{3}{2} \pi \gamma\right)\right]\right\} \\
& \times \cos \left\{\omega_{0} t+\varphi_{0}+\varepsilon æ t\left(\omega_{0}\right)^{\gamma-1}\left[\cos \left(\frac{1}{2} \pi \gamma\right)+\frac{1}{2} \varepsilon æ(2 \gamma-1)\left(\omega_{0}\right)^{\gamma-2} \cos \pi \gamma\right.\right. \\
& \left.\left.+\frac{1}{2} \varepsilon^{2} æ^{2}(1-\gamma)(1-3 \gamma)\left(\omega_{0}\right)^{2(\gamma-2)} \cos \left(\frac{3}{2} \pi \gamma\right)\right]\right\} .
\end{aligned}
$$


Equation (29a) can be rewritten as

$$
\begin{aligned}
x= & \varepsilon a_{0} e^{-\alpha t} \cos \left(\omega t+\varphi_{0}\right), \\
\alpha= & \varepsilon \rightsquigarrow\left(\omega_{0}\right)^{\gamma-1} \sin \left(\frac{1}{2} \pi \gamma\right)+\frac{1}{2} \varepsilon^{2} æ^{2}(2 \gamma-1)\left(\omega_{0}\right)^{2 \gamma-3} \sin \pi \gamma \\
& +\frac{1}{2} \varepsilon^{3} æ^{3}(1-\gamma)(1-3 \gamma)\left(\omega_{0}\right)^{3 \gamma-5} \sin \left(\frac{3}{2} \pi \gamma\right), \\
\omega= & \omega_{0}+\varepsilon æ\left(\omega_{0}\right)^{\gamma-1} \cos \left(\frac{1}{2} \pi \gamma\right)+\frac{1}{2} \varepsilon^{2} æ^{2}(2 \gamma-1)\left(\omega_{0}\right)^{2 \gamma-3} \cos \pi \gamma \\
& +\frac{1}{2} \varepsilon^{3} æ^{3}(1-\gamma)(1-3 \gamma)\left(\omega_{0}\right)^{3 \gamma-5} \cos \left(\frac{3}{2} \pi \gamma\right) .
\end{aligned}
$$

Reference to Eqs (29c) and (29d) shows that

$$
\frac{\alpha}{\omega-\omega_{0}} \approx \tan \left(\frac{1}{2} \pi \gamma\right)
$$

i.e., the coefficient of dissipation depends approximately linearly on the difference of frequencies.

When $\gamma \rightarrow 1$, Eq. (29a) goes over into the equation describing vibrations of a viscoelastic oscillator with ordinary Kelvin-Voight constitutive relations, i.e.,

$$
x=\varepsilon a_{0} e^{-\varepsilon æ t} \cos \left(\omega_{0} t+\varphi_{0}-\frac{1}{2} \varepsilon^{2} æ^{2} \omega_{0}^{-1} t\right),
$$

in so doing

$$
\omega-\omega_{0}=-\frac{1}{2} \alpha^{2} \omega_{0}^{-1}
$$

Note that Eq. (4a), which is similar to Eq. (11) if $\gamma=1 / 2$, was studied by the method of averaging by Wahi and Chatterjee in [26], where the authors compared in Fig. 2 their calculations for the envelope of the amplitude with the exact solution via Laplace transform citing the monograph [35] and declared the excellent matching. But the authors of this paper could not find in the book by Oldham and Spanier [55] even mentioning an equation similar to Eq. (4a). That is why from the text in page 10 of [26] it is not clear whose solution via Laplace transform was taken and presented in Fig. 2 in [26] for the comparison purpose, since previously many researchers studied the fractional derivative Kelvin-Voigt oscillator at $\gamma=1 / 2$ without the assumption of small fractional damping utilizing the Laplace transformation technique [56-59] including the authors of the given paper [1,13]. Note that the solution for the case of small viscosity can be obtained from the exact relationship for the Green function for this oscillator at small magnitudes of the retardation time.

\section{Free damped vibrations of a linear oscillator in a medium with finite viscosity}

Equation of motion of such an oscillator has the form

$$
\ddot{x}+\omega_{0}^{2} x+x\left(\frac{d}{d t}\right)^{\gamma} x=0 .
$$

The solution of Eq. (31) is sought in the form

$$
x=C e^{p t},
$$

where $C$ is an arbitrary constant, and $p$ is yet undetermined constant. 
Substituting the proposed solution Eq. (32) in Eq. (31) and considering Eq. (9), we are led to the characteristic equation for determining the magnitude of $p$

$$
p^{2}+æ p^{\gamma}+\omega_{0}^{2}=0 .
$$

For finding the roots of Eq. (33), we shall consider the value $æ$ as an unknown variable which is defined from Eq. (33) together with its roots.

Representing the roots of Eq. (33) in the form

$$
p=r e^{i \psi},
$$

and substituting Eq. (34) in Eq. (33), after separating the real and imaginary parts, we obtain

$$
\begin{aligned}
& r^{2} \cos 2 \psi+æ r^{\gamma} \cos \gamma \psi+\omega_{0}^{2}=0, \\
& r^{2} \sin 2 \psi+æ r^{\gamma} \sin \gamma \psi=0 .
\end{aligned}
$$

When $|\psi|<\frac{\pi}{2}$ and at $\psi= \pm \pi$, the set of Eq. (35) is rootless, since Eq. (35b) at those magnitudes of $\psi$ is not vanished to zero anywhere. In other words, all roots locate in the left half-plane, and there are no real negative roots.

For calculating complex conjugate roots of Eqs (35a) and (35b), when $\frac{1}{2} \pi<|\psi|<\pi$, let us introduce new variables, namely: $x_{1}=r^{2}$, and $x_{2}=æ r^{\gamma}$. Then for each fixed angle $\psi$ at the given $\gamma$ and $\omega_{0}^{2}$ we obtain the following set of equations in $x_{1}$ and $x_{2}$ :

$$
\begin{aligned}
& x_{1} \cos 2 \psi+x_{2} \cos \gamma \psi=-\omega_{0}^{2}, \\
& x_{1} \sin 2 \psi+x_{2} \sin \gamma \psi=0 .
\end{aligned}
$$

From Eqs (36a) and (36b) we find at each fixed magnitude of $|\psi|$

$$
x_{1}=\omega_{0}^{2} \frac{\sin \gamma \psi}{\sin (2-\gamma) \psi}, \quad x_{2}=-\omega_{0}^{2} \frac{\sin 2 \psi}{\sin (2-\gamma) \psi},
$$

and knowing now $x_{1}$ and $x_{2}$ we first determine $r=x_{1}^{1 / 2}$, and then calculate $æ=x_{2} r^{-\gamma}$.

Thus, as it follows from Eq. (37), the characteristic Eq. (33) possesses two complex conjugate roots. To investigate the asymptotic behavior of the characteristic equation roots when $æ \rightarrow \infty$ and $æ \rightarrow 0$, we put $æ=\omega_{0}^{2} \tau_{\sigma}^{\gamma}$, where $\tau_{\sigma}$ is the retardation time, and rewrite Eq. (33) in the form

$$
p^{2}\left[\left(p \tau_{\sigma}\right)^{\gamma}+1\right]^{-1}+\omega_{0}^{2}=0
$$

Considering Eq. (34), let us represent the expression $1+\left(p \tau_{\sigma}\right)^{\gamma}$ in the geometrical form, i.e.,

$$
\begin{aligned}
& 1+\left(p \tau_{\sigma}\right)^{\gamma}=R e^{i \Phi}, \\
& R=\sqrt{1+2\left(r \tau_{\sigma}\right)^{\gamma} \cos \gamma \psi+\left(r \tau_{\sigma}\right)^{2 \gamma}}, \quad \tan \Phi=\frac{\left(r \tau_{\sigma}\right)^{\gamma} \sin \gamma \psi}{1+\left(r \tau_{\sigma}\right)^{\gamma} \cos \gamma \psi} .
\end{aligned}
$$

With due account for (39a), Eq. (38) takes the form

$$
r^{2} R^{-1} e^{i(2 \psi-\Phi)}+\omega_{0}^{2}=0 .
$$

Separating the real and imaginary parts in Eq. (40) yields

$$
\begin{aligned}
& r^{2} R^{-1} \cos (2 \psi-\Phi)+\omega_{0}^{2}=0 . \\
& r^{2} R^{-1} \sin (2 \psi-\Phi)=0 .
\end{aligned}
$$


From Eqs (41a) and (41b) we find

$$
\begin{aligned}
& 2 \psi-\Phi= \pm \pi \\
& r^{2} R^{-1}=\omega_{0}^{2} .
\end{aligned}
$$

Tending in expressions (39b) and (42) the value of $\tau_{\sigma}$ to 0 and $\infty$, we have

$$
\begin{aligned}
& r_{0}=\omega_{0}, \quad \psi_{0}= \pm \frac{\pi}{2} \\
& r_{\infty}=\infty, \quad \psi_{\infty}= \pm \frac{\pi}{2-\gamma} .
\end{aligned}
$$

Reference to Eqs (44) and (45) shows that the curves characterizing the behavior of the complex conjugate roots of Eq. (33) on the complex plane $p$ as the function of the parameter $æ$ have asymptotes leaving the origin of the coordinates under the angles $\psi= \pm \pi /(2-\gamma)$ to the real semi-axis.

The behavior of the roots

$$
p_{1,2}=r e^{ \pm i \psi}=-\alpha \pm i \omega
$$

of Eq. (33) as function of the parameter æ, which varies from 0 to $+\infty$, is shown in Fig. 1 at $\omega_{0}^{2}=1$, where the magnitudes of the value $\gamma$ are indicated by figures. It is seen that as the value $æ$ changes from 0 to $+\infty$, the curves for the æ-dependence of the roots $p_{1,2}$ (solid curves in Fig. 1) issue out of the points $\pm i$ for all magnitudes $0<\gamma<1$, and then grow and tend to $+\infty$ as $æ \rightarrow+\infty$, in so doing rapidly come close to the corresponding asymptotes (dashed lines in Fig. 1). As this takes place, they never intersect the real negative semi-axis and locate in the left half-plane of the complex plane $p$. When $\gamma \rightarrow 1$, the roots of the characteristic Eq. (33) tend to the roots of the characteristic equation for the conventional Kelvin-Voight model, i.e., at $\omega_{0}=1$ they are determined by the formula

$$
p_{1,2}=-\frac{1}{2} æ \pm \sqrt{\frac{1}{4} æ^{2}-1},
$$

whence it is evident that the roots remain to be complex conjugate with variation of $æ$ from 0 to 2 , while for $æ 2$ the roots become real negative ones (the domain of aperiodicity).

From the asymptotic behavior of the roots it follows that if $\omega \gg \omega_{0}$, then

$$
\frac{\alpha}{\omega-\omega_{0}} \approx \frac{\alpha}{\omega}=\tan \left(\frac{1}{2} \pi \frac{\gamma}{2-\gamma}\right) .
$$

A comparison of Eqs (30) and (46c) shows that $\alpha$ and $\omega-\omega_{0}$ are proportional to each other in both cases, although the coefficients of proportionality differ a little, what is explained by the fact that in the first case viscosity was assumed to be small, while in the second case it is finite.

Knowing the behavior of the roots of the characteristic Eq. (33), the solution of Eq. (31) is written as

$$
x(t)=C_{1} e^{(-\alpha+i \omega) t}+C_{2} e^{(-\alpha-i \omega) t}=a_{0} e^{-\alpha t} \cos \left(\omega t+\varphi_{0}\right),
$$

where $a_{0}$ and $\varphi_{0}$ are the constants to be determined from the initial conditions

$$
x(0)=x_{0}, \quad \dot{x}(0)=v_{0} .
$$

Equation (47a) describes free damped vibrations of the oscillator and is not dissimilar in aspect to related formula for the conventional Kelvin-Voigt model. However, there exists the fundamental difference between them. The matter is fact that in the domain of aperiodic motion the roots of the characteristic equation for the conventional Kelvin-Voigt model become real ones. As this takes place, the oscillatory regime is changed by the aperiodic motion such that $x(t)$ decays monotonically to zero. As for the roots of the characteristic equation for the fractional derivative Kelvin-Voigt model, then they remain to be complex conjugate at any magnitude of the parameter $æ$, that is the principal possibility for transition of such a system into the aperiodic regime is absent $[1,13,60]$. This fact has been verified experimentally in [61].

From the comparison of Eq. (47) with the solution Eq. (29) it can be noted that in the latter the ratio of the damping coefficient to the difference in frequencies remains constant for any value of the parameter $æ$, while in this problem this ratio could be considered as constant beginning from a certain magnitude of the parameter æ, moreover the magnitude of $æ$ takes on its own value for each fixed $\gamma$. 


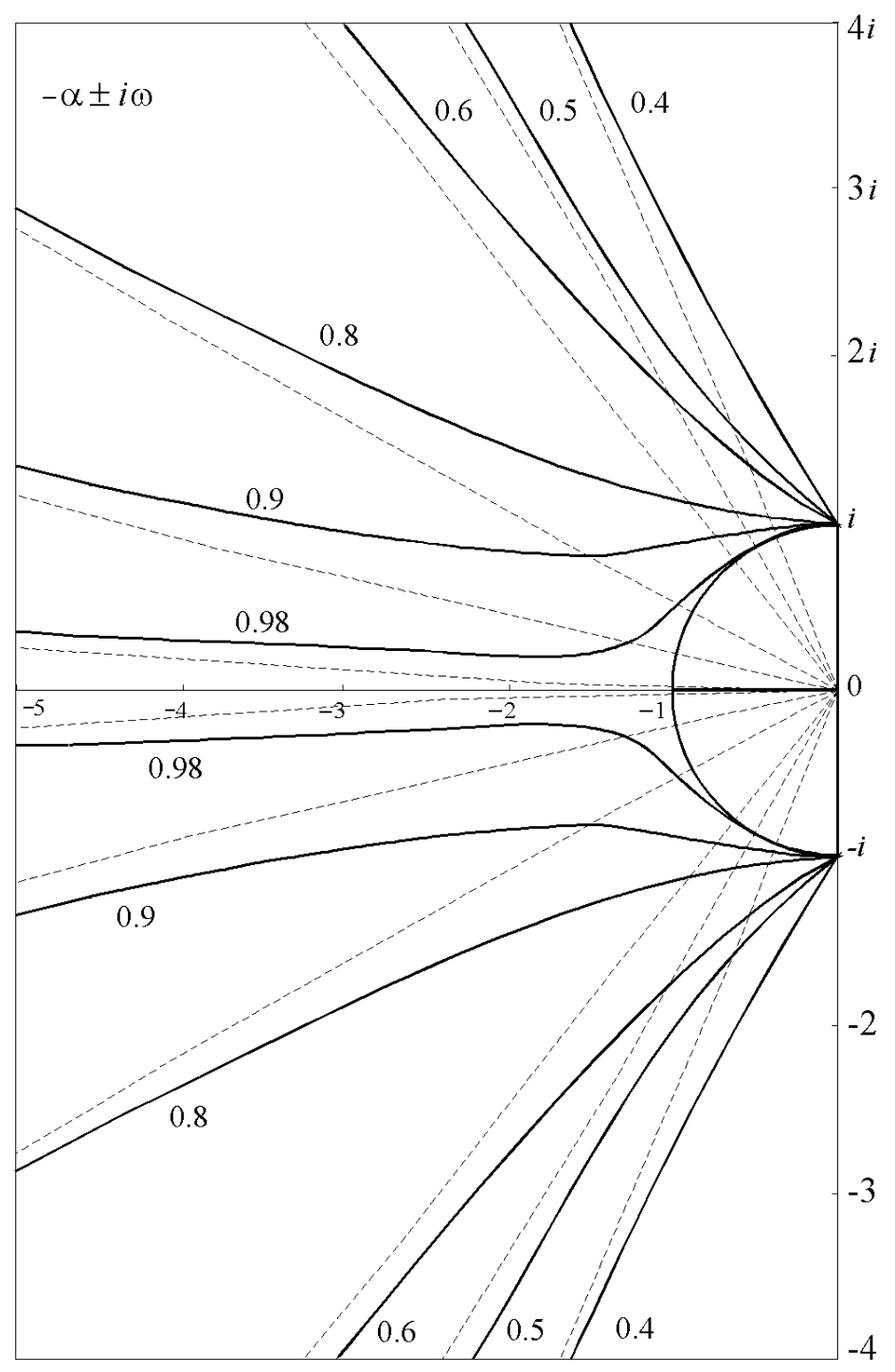

Fig. 1. The behavior of the complex conjugate roots for the Kelvin-Voight fractional oscillator.

\section{Free damped vibrations of a linear oscillator with fractional derivative in the inertial force description in a medium with fractional viscosity}

As it has been already mentioned in Introduction, the application of 'fractional force of inertia' in equations of motion of mechanical systems is unjustified from the point of view of engineering practice. Let us demonstrate the validity of our statement analysing the simplest oscillators as example.

For this purpose, let us change the second time-derivative in Eq. (31) by the derivative of noninteger order. The equations of motion of such oscillators could be written in the form

$$
\left(\frac{d}{d t}\right)^{1+\beta} x+\omega_{0}^{1+\beta} x+æ_{1}\left(\frac{d}{d t}\right)^{\gamma} x=0
$$

or 


$$
\left(\frac{d}{d t}\right)^{2-\beta} x+\omega_{0}^{2-\beta} x+æ_{2}\left(\frac{d}{d t}\right)^{\gamma} x=0,
$$

where $æ_{1}=\omega_{0}^{1+\beta} \tau_{\sigma}^{\gamma}, æ_{2}=\omega_{0}^{2-\beta} \tau_{\sigma}^{\gamma}$, and $0<\beta, \gamma<1$.

Implementing the substitution (32) in Eqs (48a) and (48b), we are led, respectively, to the following characteristic equations:

$$
\begin{aligned}
& p^{1+\beta}+æ_{1} p^{\gamma}+\omega_{0}^{1+\beta}=0 \\
& p^{2-\beta}+æ_{2} p^{\gamma}+\omega_{0}^{2-\beta}=0 .
\end{aligned}
$$

At first glance it is expected that the solutions of Eqs (48a) and (48b) should be the same as the fractional parameter $\beta$ varies from 0 to 1 in the both models. But it is not the case, since the roots of the corresponding characteristic Eqs (49a) and (49b) at the same magnitudes of $\beta$ and $\gamma$ behave in a different way, what will be demonstrated below.

\subsection{Analysis of the oscillator with the constitutive Eq. (48a)}

Representing the roots of Eq. (49a) in the form of Eq. (34), from Eq. (49a) we find

$$
\begin{aligned}
& r^{1+\beta} \cos (1+\beta) \psi+æ_{1} r^{\gamma} \cos \gamma \psi+\omega_{0}^{1+\beta}=0, \\
& r^{1+\beta} \sin (1+\beta) \psi+æ_{1} r^{\gamma} \sin \gamma \psi=0 .
\end{aligned}
$$

At $|\psi| \leq \frac{1}{2} \pi$, the set of Eq. (50) has no roots, since Eq. (50b) does not vanish to zero at these magnitudes of $\psi$, i.e., all roots locate in the left half-plane of the complex plane $p$. To show the absence of real roots for Eq. (49a), let us put in it $p=-y, y>0$ and divide the real and imaginary parts. As a result we obtain

$$
\begin{aligned}
& -y^{1+\beta} \cos \pi \beta+æ_{1} y^{\gamma} \cos \pi \gamma+\omega_{0}^{1+\beta}=0, \\
& -y^{1+\beta} \sin \pi \beta+æ_{1} y^{\gamma} \sin \pi \gamma=0,
\end{aligned}
$$

whence it follows

$$
y^{\beta}=-\frac{\omega_{0}^{1+\beta} \sin \pi \beta}{\mathfrak{1}_{1} \sin (\beta-\gamma) \pi} .
$$

If we suppose that $\beta>\gamma$, then relationship (51) is contradictory. As it will be shown below, the assumption $\beta>\gamma$ is not unreasonable.

To calculate the complex conjugate roots of the set of Eq. (50) at $\frac{1}{2} \pi<|\psi|<\pi$, let us introduce new variables, namely: $x_{1}=r^{1+\beta}$, and $x_{2}=æ_{1} r^{\gamma}$. Then for each fixed angle $\psi$ at the given $\gamma, \beta$, and $\omega_{0}$ we obtain the following set of equations in $x_{1}$ and $x_{2}$ :

$$
\begin{aligned}
& x_{1} \cos (1+\beta) \psi+x_{2} \cos \gamma \psi=-\omega_{0}^{1+\beta}, \\
& x_{1} \sin (1+\beta) \psi+x_{2} \sin \gamma \psi=0 .
\end{aligned}
$$

From Eq. (52) for each fixed magnitude of $|\psi|$ we find

$$
x_{1}=\omega_{0}^{1+\beta} \frac{\sin \gamma \psi}{\sin (1+\beta-\gamma) \psi}, \quad x_{2}=-\omega_{0}^{1+\beta} \frac{\sin (1+\beta) \psi}{\sin (1+\beta-\gamma) \psi},
$$

and now knowing $x_{1}$ and $x_{2}$ we first determine $r=x_{1}^{\frac{1}{1+\beta}}$, and then $æ_{1}=x_{2} r^{-\gamma}$. 
Thus, as it follows from Eq. (53), the characteristic Eq. (49a) possesses two complex conjugate roots. To analyse their asymptotic behavior at $æ_{1} \rightarrow \infty\left(\tau_{\sigma} \rightarrow \infty\right)$ and $æ_{1} \rightarrow 0\left(\tau_{\sigma} \rightarrow 0\right)$, we rewrite Eq. (49a) as

$$
p^{1+\beta}\left[\left(p \tau_{\sigma}\right)^{\gamma}+1\right]^{-1}+\omega_{0}^{1+\beta}=0 .
$$

Considering Eqs (34) and (39), Eq. (54) can be rewritten in the form

$$
r^{1+\beta} R^{-1} e^{i[(1+\beta) \psi-\Phi]}+\omega_{0}^{1+\beta}=0 .
$$

Separating in Eq. (55) the real and imaginary parts yields

$$
\begin{aligned}
& r^{1+\beta} R^{-1} \cos [(1+\beta) \psi-\Phi]+\omega_{0}^{1+\beta}=0, \\
& r^{1+\beta} R^{-1} \sin [(1+\beta) \psi-\Phi]=0,
\end{aligned}
$$

whence it follows that

$$
\begin{aligned}
& (1+\beta) \psi-\Phi= \pm \pi, \\
& r^{1+\beta} R^{-1}=\omega_{0}^{1+\beta} .
\end{aligned}
$$

Tending the value $\tau_{\sigma}$ to 0 and $\infty$ in Eqs (39b) and (57), we have

$$
\begin{aligned}
& r_{0}=\omega_{0}, \quad \psi_{0}= \pm \frac{\pi}{1+\beta} \quad\left(\tau_{\sigma} \rightarrow 0\right), \\
& r_{\infty}^{\frac{1+\beta-\gamma}{1+\beta}} \approx \omega_{0}\left(\tau_{\sigma}\right)^{\frac{\gamma}{1+\beta}} \rightarrow \infty, \quad \psi_{\infty}= \pm \frac{\pi}{1+\beta-\gamma} \quad\left(\tau_{\sigma} \rightarrow \infty\right) .
\end{aligned}
$$

The limiting angles $\psi_{0}$ and $\psi_{\infty}$ in Eq. (58) should locate within the limits from $\frac{1}{2} \pi$ to $\pi$. For the angle $\psi_{\infty}$, this is possible only in the case if $\beta \geqslant \gamma$.

Reference to Eq. (58b) shows that the curves, which characterize the behavior of the complex conjugate roots of Eq. (49a) in the complex plane as the function of the parameter $æ_{1}$, possess the asymptotes issuing out from the origin of the coordinates under the angles $\psi_{\infty}= \pm \pi /(1+\beta-\gamma)$ to the real axis.

\subsection{Analysis of the oscillator with the constitutive Eq. (48b)}

It can be shown in a similar way that the characteristic Eq. (49b) lacks real negative roots at $0<\beta+\gamma \leq 1$, since for $p=-y, y>0$ we are led to the contradictory equality

$$
y^{\beta}=-\frac{\omega_{0}^{2-\beta} \sin \pi \beta}{æ_{2} \sin (\beta+\gamma) \pi} .
$$

Equation (49b) possesses two complex conjugate roots locating in the left half-plane of the complex plane $p$, which take on the following limiting magnitudes:

$$
\begin{aligned}
& r_{0}=\omega_{0}, \quad \psi_{0}= \pm \frac{\pi}{2-\beta} \quad\left(\tau_{\sigma} \rightarrow 0\right), \\
& r_{\infty}^{\frac{2-\beta-\gamma}{2-\beta}} \approx \omega_{0}\left(\tau_{\sigma}\right)^{\frac{\gamma}{2-\beta}} \rightarrow \infty, \quad \psi_{\infty}= \pm \frac{\pi}{2-\beta-\gamma} \quad\left(\tau_{\sigma} \rightarrow \infty\right) .
\end{aligned}
$$

If the angle $\psi_{\infty}$ lies within the domain of $\frac{\pi}{2} \leq \psi_{\infty} \leq \pi$, then from Eq. (59b) it follows that $\beta+\gamma \leq 1$. Such a constraint restricts significantly the domain of applicability of the model (48b), as compared with the model (48a), if, of course, one takes no account for physical meaning of "fractional force of inertia" in the both models.

Reference to Eq. (59b) shows that the curves, which characterize the behavior of the complex conjugate roots of Eq. (49b) in the complex plane as the function of the parameter $x_{2}$, possess the asymptotes issuing out from the origin of the coordinates under the angles $\psi_{\infty}= \pm \pi /(2-\beta-\gamma)$ to the real axis. 


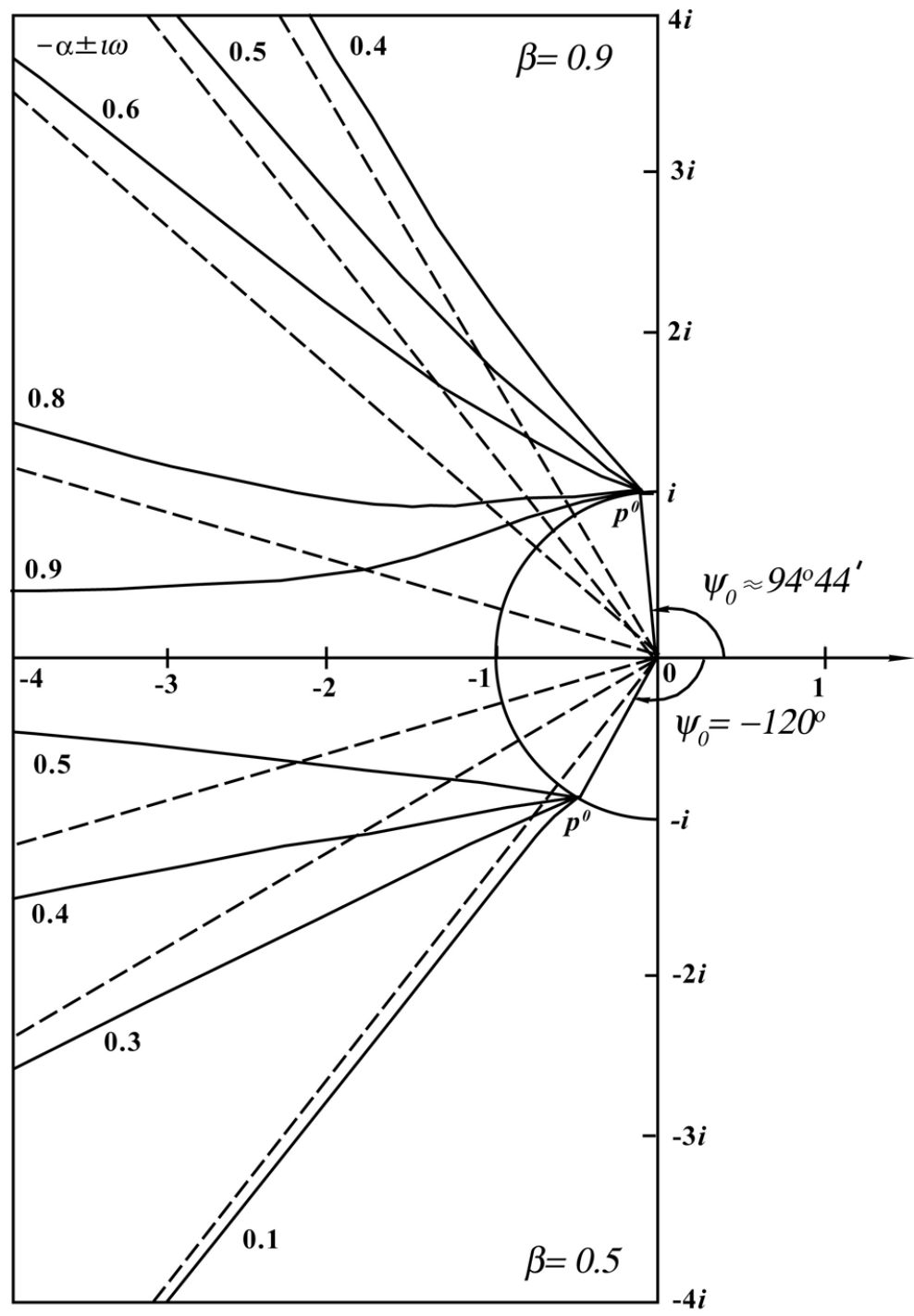

(a)

Fig. 2. The behavior of the complex conjugate roots for the fractional oscillator: (a) model (48a) and (b) model (48b).

\subsection{Comparative analysis of the characteristic equation roots behavior in the complex plane $p$}

The behavior of the roots of Eqs (49a) and (49b) as function of the parameter $æ_{1}$ for Eq. (49a) and $æ_{2}$ for Eq. (49b) during their variation from 0 to $+\infty$ for $\omega_{0}=1$ is shown in the complex plane $p$ in Fig. 2a and 2b, respectively. The magnitudes of the fractional parameter $\gamma$ are indicated by figures near the corresponding curves. The curves are presented only for one complex conjugate root, in so doing the curves in the upper left quarter of the complex plane correspond to the cases $\beta=0.9$ (Fig. 2a) and $\beta=0.4$ (Fig. 2b), while those in the lower quarter of the complex plane are constructed at $\beta=0.5$ (Fig. 2a) and $\beta=0.6$ (Fig. 2b).

From Fig. $2 \mathrm{a}$ and $2 \mathrm{~b}$ it is evident that as $æ_{1}$ or $æ_{2}$ varies from 0 to $+\infty$, the curves of the $æ_{1}$-dependence of $p_{1,2}$ (solid curves in Fig. 2) issue out from the points $p_{1,2}^{0}=\omega_{0} \exp \left( \pm i \frac{\pi}{1+\beta}\right)$, when $æ_{1}=0$, at all magnitudes of $0<\beta, \gamma<1 \quad(\beta \geqslant \gamma)$ in Fig. $2 \mathrm{a}$ and the curves of the $x_{2}$-dependence of $p_{1,2}$ issue out from the points 


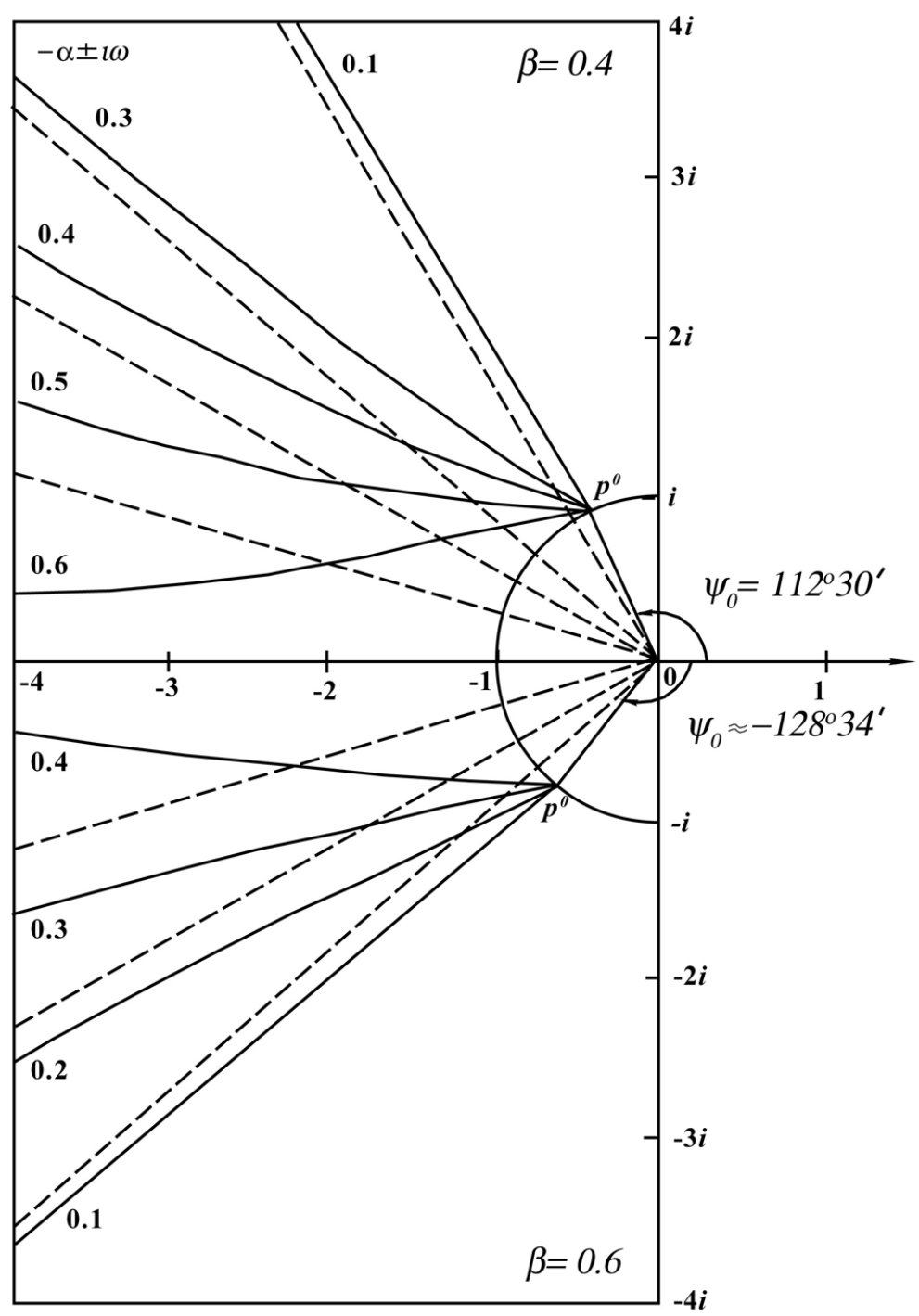

(b)

Fig. 2, continued.

$p_{1,2}^{0}=\omega_{0} \exp \left( \pm i \frac{\pi}{2-\beta}\right)$, when $æ_{2}=0$, at all magnitudes of $0<\beta, \gamma<1 \quad(\beta+\gamma \leq 1)$ in Fig. 2b. Further all curves tend to $+\infty$ when $æ_{1}$ or $æ_{2} \rightarrow+\infty$, in so doing approaching the corresponding asymptotes (dashed lines in Fig. 2). As this takes place, the curves do not intersect the real negative semi-axis anywhere, i.e., the possibility for the oscillator to go into an aperiodic regime is absent, and locate in the left half-plane of the complex plane $p$.

The roots of Eq. (49a), when $\beta \rightarrow 1$, and those of Eq. (49b), when $\beta \rightarrow 0$, go over in the roots of Eq. (33) presented in Fig. 1.

At first glance it seems likely that the behavior of the roots in Fig. $2 a$ and $2 b$ for the oscillators with fractional derivatives in the inertial force description differes little from that in Fig. 1 for the oscillator with conventional inertial force, with the exception of the initial points $p_{1,2}^{0}$ for the constructed curves: in the given problems, these points are complex ones, what corresponds to the damping oscillation regime, while in the previous problem these points belong to the imaginary axis, what is in compliance with undamped oscillation regime. But the initial points of the curves are obtained at $\tau_{\sigma}=0$, i.e., at these points the retardation (creep) time is equal to zero, and hence oscillatory 
process should occur without energy dissipation. In other words, in the problem under consideration in this Section, the physical meaning of the parameters involving in the equation of motion of the oscillator is wrenched, resulting in erroneous conclusions concerning its behavior.

Of course, such a contradiction in the behavior of the models (48a) and (48b) may be treated as the existence of the 'intrinsic damping characteristic of the fractional oscillator', which is governed by the fractional parameter $\beta$ (at $\tau_{\sigma}=0$ the given models goes over into the elastic model only at $\beta=1$ and $\beta=0$, respectively, see Fig. 1). Such an interpretation of the fractional force of inertia has been proposed in $[8,62,63]$, and even has found some followers [64]. However, this has little force for the engineer, since it remains a mystery how to determine experimentally the magnitude of the parameter $\beta$ in this case, i.e., how to calibrate 'fractional' inertia.

\section{Vibrations of a nonlinear oscillator in a medium with small viscosity}

\subsection{Viscosity of the order of $\varepsilon$}

Let us consider free damped vibrations of a nonlinear oscillator, the equation of motion of which has the form

$$
\ddot{x}+\omega_{0}^{2} x+k x^{3}+2 \varepsilon æ\left(\frac{d}{d t}\right)^{\gamma} x=0,
$$

where $k$ is an elastic coefficient of nonlinear stiffness per unit mass.

To construct the solution of Eq. (60), we shall use the method of multiple time scales [54]. Considering Eqs (12) and (13), and limiting ourselves to the terms of the order of $\varepsilon^{4}$, as a result we are led to the set of recurrent equations, first two of which coincide with Eq. (14a) and (14b), while the other two have the form:

$$
\begin{aligned}
D_{0}^{2} x_{3}+\omega_{0}^{2} x_{3}= & -2 æ D_{+}^{\gamma} x_{2}-2 D_{0} D_{1} x_{2}-2 æ \gamma D_{+}^{\gamma-1} D_{1} x_{1}-\left(D_{1}^{2}+2 D_{0} D_{2}\right) x_{1}-k x_{1}^{3}, \\
D_{0}^{2} x_{4}+\omega_{0}^{2} x_{4}= & -2 æ D_{+}^{\gamma} x_{3}-2 D_{0} D_{1} x_{3}-2 æ \gamma D_{+}^{\gamma-1} D_{1} x_{2}-\left(D_{1}^{2}+2 D_{0} D_{2}\right) x_{2} \\
& -æ \gamma\left[(\gamma-1) D_{+}^{\gamma-2} D_{1}^{2}+2 D_{+}^{\gamma-1} D_{2}\right] x_{1}-2\left(D_{0} D_{3}+D_{1} D_{2}\right) x_{1}-3 k x_{1}^{2} x_{2} .
\end{aligned}
$$

Equations (61a) and (61b) differ from Eqs (14c) and (14d), respectively, by their last terms describing cubic nonlinearity of the oscillator.

Carrying out the procedure described in detail in Section 2, one can find

$$
\begin{aligned}
x_{1}\left(T_{0}, T_{1}, T_{2}, T_{3}\right)= & a_{111} \exp \left(i \omega_{0} T_{0}\right) \exp \left[-\left(i \omega_{0}\right)^{\gamma-1} æ T_{1}\right] \exp \left[\frac{1}{2} \mathfrak{x}^{2}\left(i \omega_{0}\right)^{2 \gamma-3}(2 \gamma-1) T_{2}\right] \\
& \times \exp \left[-\frac{1}{2} \Re^{3}\left(i \omega_{0}\right)^{3 \gamma-5}(1-\gamma)(1-3 \gamma) T_{3}\right]+c c, \\
x_{2}\left(T_{0}, T_{1}, T_{2}, T_{3}\right)= & a_{222} \exp \left(i \omega_{0} T_{0}\right) \exp \left[-\left(i \omega_{0}\right)^{\gamma-1} æ T_{1}\right] \exp \left[\frac{1}{2} \Re^{2}\left(i \omega_{0}\right)^{2 \gamma-3}(2 \gamma-1) T_{2}\right] \\
& \times \exp \left[-\frac{1}{2} \Re^{3}\left(i \omega_{0}\right)^{3 \gamma-5}(1-\gamma)(1-3 \gamma) T_{3}\right] \\
& -i a_{111}^{3} \frac{3 k}{4 \sin \left(\frac{\pi \gamma}{2}\right)\left(\omega_{0}\right)^{\gamma-2}} \\
& \times \exp \left(i \omega_{0} T_{0}\right) \exp \left[-\left(\omega_{0}\right)^{\gamma-1} æ\left(3 \sin \left(\frac{\pi \gamma}{2}\right)-i \cos \left(\frac{\pi \gamma}{2}\right)\right) T_{1}\right] \\
& \times \exp \left[\frac{1}{2} \Re^{2}\left(\omega_{0}\right)^{2 \gamma-3}(2 \gamma-1)(3 \sin \pi \gamma-i \cos \pi \gamma) T_{2}\right]
\end{aligned}
$$




$$
\begin{aligned}
& \times \exp \left[-\frac{1}{2} \mathfrak{x}^{3}\left(\omega_{0}\right)^{3 \gamma-5}(1-\gamma)(1-3 \gamma)\left(3 \sin \left(\frac{3 \pi \gamma}{2}\right)-i \cos \left(\frac{3 \pi \gamma}{2}\right)\right) T_{3}\right] \\
& +c c .
\end{aligned}
$$

Thus, the solution for the nonlinear oscillator (60) can be written as

$$
\begin{aligned}
x= & x^{\text {lin.os. }}+\varepsilon^{2} a_{111}^{3} \frac{3 k}{4 \sin \left(\frac{\pi \gamma}{2}\right)\left(\omega_{0}\right)^{\gamma-2}} \exp \left\{-3 \varepsilon æ t\left(\omega_{0}\right)^{\gamma-1}\left[\sin \left(\frac{1}{2} \pi \gamma\right)\right.\right. \\
& \left.\left.+\frac{1}{2} \varepsilon æ(2 \gamma-1)\left(\omega_{0}\right)^{\gamma-2} \sin \pi \gamma+\frac{1}{2} \varepsilon^{2} æ^{2}(1-\gamma)(1-3 \gamma)\left(\omega_{0}\right)^{2(\gamma-2)} \sin \left(\frac{3}{2} \pi \gamma\right)\right]\right\} \\
& \times \sin \left\{\omega_{0} t+\varepsilon æ t\left(\omega_{0}\right)^{\gamma-1}\left[\cos \left(\frac{1}{2} \pi \gamma\right)+\frac{1}{2} \varepsilon æ(2 \gamma-1)\left(\omega_{0}\right)^{\gamma-2} \cos \pi \gamma\right.\right. \\
& \left.\left.+\frac{1}{2} \varepsilon^{2} æ^{2}(1-\gamma)(1-3 \gamma)\left(\omega_{0}\right)^{2(\gamma-2)} \cos \left(\frac{3}{2} \pi \gamma\right)\right]\right\}
\end{aligned}
$$

where $a_{111}$ and $a_{222}$ are real constants determined by the initial conditions, and $x^{\text {lin.os. }}$ is the linear part of the solution coinciding with that for a linear elastic oscillator (29a) vibrating in a viscoelastic medium with the same type of viscosity.

When $\gamma \rightarrow 1$, Eq. (63) goes over into the equation describing vibrations of a nonlinear viscoelastic oscillator with conventional Kelvin-Voight constitutive relations, i.e.,

$$
\begin{aligned}
x= & 2 \varepsilon\left(a_{111}+\varepsilon a_{222}\right) e^{-\varepsilon æ t} \cos \left(\omega_{0} t-\frac{1}{2} \varepsilon^{2} æ^{2} \omega_{0}^{-1} t\right) \\
& +\frac{3}{4} \varepsilon^{2} a_{111}^{3} \omega_{0} e^{-3 \varepsilon æ t} \sin \left(\omega_{0} t-\frac{1}{2} \varepsilon^{2} æ^{2} \omega_{0}^{-1} t\right) .
\end{aligned}
$$

5.2. Viscosity of the order of $\varepsilon^{2}$

Now let us consider vibrations of a nonlinear oscillator, the equation of motion of which has the form

$$
\ddot{x}+\omega_{0}^{2} x+k x^{3}+\varepsilon^{2} x\left(\frac{d}{d t}\right)^{\gamma} x=0 .
$$

Note that the equation similar to Eq. (64) but involving quadratic nonlinearity has been studied in [65].

To construct the solution of Eq. (64), we shall use the method of multiple time scales [54]. Considering Eqs (12) and (13), and limiting ourselves to the terms of the order of $\varepsilon^{3}$, as a result we are led to the following set of recurrent equations:

$$
\begin{aligned}
& D_{0}^{2} x_{1}+\omega_{0}^{2} x_{1}=0 \\
& D_{0}^{2} x_{2}+\omega_{0}^{2} x_{2}=-2 D_{0} D_{1} x_{1}, \\
& D_{0}^{2} x_{3}+\omega_{0}^{2} x_{3}=-2 D_{0} D_{1} x_{2}-\left(D_{1}^{2}+2 D_{0} D_{2}\right) x_{1}-k x_{1}^{3}-æ D_{+}^{\gamma} x_{1} .
\end{aligned}
$$

The general solution of Eq. (65a) is determined by relationship (15). Substituting Eq. (15) into the right-hand side of Eq. (65b), we find

$$
D_{0}^{2} x_{2}+\omega_{0}^{2} x_{2}=-2 i \omega_{0} D_{1} A_{1} \exp \left(i \omega_{0} T_{0}\right)+c c \text {. }
$$

To eliminate circular terms in Eq. (66), it is necessary to vanish to zero the coefficient standing at $\exp \left(i \omega_{0} T_{0}\right)$, i.e., 


$$
D_{1} A_{1}\left(T_{1}, T_{2}\right)=0,
$$

whence it follows that $A_{1}$ is $T_{1}$-independent.

Then the general solution of Eq. (66) has the form

$$
x_{2}=A_{2}\left(T_{1}, T_{2}\right) \exp \left(i \omega_{0} T_{0}\right)+\bar{A}_{2}\left(T_{1}, T_{2}\right) \exp \left(-i \omega_{0} T_{0}\right) .
$$

Substituting Eqs (15) and (68) in the right-hand side of Eq. (65c) yields

$$
\begin{aligned}
D_{0}^{2} x_{3}+\omega_{0}^{2} x_{3}= & -2 i \omega_{0} D_{1} A_{2} \exp \left(i \omega_{0} T_{0}\right)-k A_{1}^{3} \exp \left(3 i \omega_{0} T_{0}\right) \\
& -\left[2 i \omega_{0} D_{2} A_{1}+æ\left(i \omega_{0}\right)^{\gamma} A_{1}+3 k A_{1}^{2} \bar{A}_{1}\right] \exp \left(i \omega_{0} T_{0}\right)+c c .
\end{aligned}
$$

To eliminate circular terms from Eq. (69), the coefficients at $\exp \left(i \omega_{0} T_{0}\right)$ should be equated to zero, i.e.,

$$
D_{1} A_{2}\left(T_{1}, T_{2}\right)=0,
$$

$$
2 i \omega_{0} D_{2} A_{1}+æ\left(i \omega_{0}\right)^{\gamma} A_{1}+3 k A_{1}^{2} \bar{A}_{1}=0
$$

From Eq. (70a) it follows that $A_{2}$ is $T_{1}$-independent.

Let us multiply Eq. (70b) by $A_{1}$ and write its complex conjugate equation. Then both equations obtained we first add to each other, and subtract one from another. As a result we find

$$
\begin{aligned}
& i\left(\bar{A}_{1} D_{2} A_{1}-A_{1} D_{2} \bar{A}_{1}\right)+2 æ \omega_{0}^{\gamma-1} A_{1} \bar{A}_{1} \cos \frac{1}{2} \pi \gamma+3 k \omega_{0}^{-1} A_{1}^{2} \bar{A}_{1}^{2}=0, \\
& i\left(\bar{A}_{1} D_{2} A_{1}+A_{1} D_{2} \bar{A}_{1}\right)+2 æ i \omega_{0}^{\gamma-1} A_{1} \bar{A}_{1} \sin \frac{1}{2} \pi \gamma=0 .
\end{aligned}
$$

Representing the function $A_{1}\left(T_{2}\right)$ in the polar form

$$
A_{1}=a \exp (i \varphi)
$$

from Eqs (71a) and (71b) we obtain

$$
\begin{aligned}
& \dot{\varphi}-\frac{1}{2} \delta-\frac{3 k}{2 \omega_{0}} a^{2}=0 \\
& \left(a^{2}\right)^{\cdot}+s a^{2}=0
\end{aligned}
$$

where an overdot denotes the $T_{2}$-derivative, $\delta=æ \omega_{0}^{\gamma-1} \cos \frac{1}{2} \pi \gamma$, and $s=æ \omega_{0}^{\gamma-1} \sin \frac{1}{2} \pi \gamma$.

Integrating Eqs (72a) and (72b) yields

$$
\begin{aligned}
& a^{2}=a_{0}^{2} e^{-s T_{2}}, \\
& \varphi=\frac{1}{2} \delta T_{2}-\frac{3 k}{2 \omega_{0} s} a_{0}^{2} e^{-s T_{2}}+\varphi_{0},
\end{aligned}
$$

where $a_{0}$ and $\varphi_{0}$ are the initial magnitudes of $a$ and $\varphi$, respectively.

Eliminating the time $T_{2}$ from Eqs (72a) and (72b), we find

$$
\varphi=-\frac{\delta}{s} \ln \frac{a}{a_{0}}-\frac{3 k}{2 \omega_{0} s}\left(a^{2}-a_{0}^{2}\right)+\varphi_{0}
$$

or

$$
G(\ln a, \varphi)=\frac{1}{2} \varphi s+\frac{1}{2} \delta \ln a+\frac{3 k}{4 \omega_{0}} \exp (2 \ln a)=G_{0}\left(\ln a_{0}, \varphi_{0}\right) .
$$


Relationship (74b) can be interpreted as the stream-function for the phase fluid moving in the plane with the coordinates $\ln a, \varphi$. Really, considering Eqs (72a) and (72b), it is followed from Eq. (74b) that the components of the vector of the phase fluid motion $\mathbf{V}\left\{v_{\ln a}=(\ln a)^{\cdot}, v_{\varphi}=\dot{\varphi}\right\}$ are determined by the following formulas:

$$
(\ln a)^{\cdot}=-\frac{\partial G}{\partial \varphi}, \quad \dot{\varphi}=\frac{\partial G}{\partial(\ln a)} .
$$

Since

$$
d G=\frac{\partial G}{\partial \varphi} d \varphi+\frac{\partial G}{\partial(\ln a)} d(\ln a)=0
$$

then with due account for Eq. (75)

$$
-v_{\ln a} d \varphi+v_{\varphi} d(\ln a)=0
$$

or

$$
\frac{d \varphi}{v_{\varphi}}=\frac{d(\ln a)}{v_{\ln a}},
$$

that is Eq. (76) is the equation of the stream-line.

The stream-lines of the phase fluid are constructed in Fig. 3a in the plane $\ln \frac{a}{a_{0}}, \varphi$ according to Eq. (74), when $\omega_{0}=1, æ=1$, and $\gamma=0.5$, for the following magnitudes of the dimensionless initial amplitude $a_{0}^{* 2}=\frac{3}{2} k a_{0}^{2}$ : $0.5,1.0,1.25$, and 1.5 for the initial phase $\varphi_{0}=1$. These lines originate from the $\varphi$-axis and locate in the domain of negative magnitudes of $\ln \frac{a}{a_{0}}$, since $a \leqslant a_{0}$. When $a \rightarrow 0$, these curves tend to $+\infty$, since the phase shift $\varphi$ increases beyond all bounds with time. As this takes place, the set of these stream-lines tend to the set of straight lines, equations of which have the form

$$
\varphi=-\cot \left(\frac{1}{2} \pi \gamma\right) \ln \frac{a}{a_{0}}+\frac{a_{0}^{* 2}}{\sin \left(\frac{1}{2} \pi \gamma\right)}+\varphi_{0}
$$

The increase in the level of the initial amplitude $a_{0}^{*}$ leads to the fact that the ordinates of the stream-lines increase at each fixed magnitude of $\ln \frac{a}{a_{0}}$. The phase fluid points move along the stream-lines with the velocity $\mathbf{V}\left\{v_{\ln a}, v_{\varphi}\right\}$ in the direction indicated by arrows.

Figure $3 \mathrm{~b}$ presents the stream-lines for the fixed magnitudes of the initial values $a_{0}^{*}=1$ and $\varphi_{0}=1$ for the different values of the fractional parameter $\gamma$, which magnitudes are indicated by figures near the corresponding curves. Reference to Fig. $3 \mathrm{~b}$ shows that the ordinates of the stream-lines increase with the decrease in the fractional parameter for each fixed magnitude of $\ln \frac{a}{a_{0}}$.

The solution for nonlinear free damped vibrations has the form

$$
\begin{aligned}
x= & \varepsilon a_{0} \exp \left(-\frac{1}{2} \varepsilon^{2} t æ \omega_{0}^{\gamma-1} \sin \frac{1}{2} \pi \gamma\right) \\
& \times \cos \left[\omega_{0} t+\varphi_{0}+\frac{1}{2} \varepsilon^{2} t æ \omega_{0}^{\gamma-1} \cos \frac{1}{2} \pi \gamma-\frac{3}{2} k a_{0}^{2}\left(æ \omega_{0}^{\gamma} \sin \frac{1}{2} \pi \gamma\right)^{-1}\right. \\
& \left.\times \exp \left(-\varepsilon^{2} t æ \omega_{0}^{\gamma-1} \sin \frac{1}{2} \pi \gamma\right)\right] .
\end{aligned}
$$

Reference to Eq. (77) shows that the ratio of the dissipation coefficient $\alpha=\frac{1}{2} \varepsilon^{2} æ \omega_{0}^{\gamma-1} \sin \frac{1}{2} \pi \gamma$ to the linear part of the difference in the frequencies $\omega-\omega_{0}=\frac{1}{2} \varepsilon^{2} æ \omega_{0}^{\gamma-1} \cos \frac{1}{2} \pi \gamma$ is defined by Eq. (30). However, the nonlinear part of $\omega-\omega_{0}$ is dependent on the squared amplitude. 
(a)

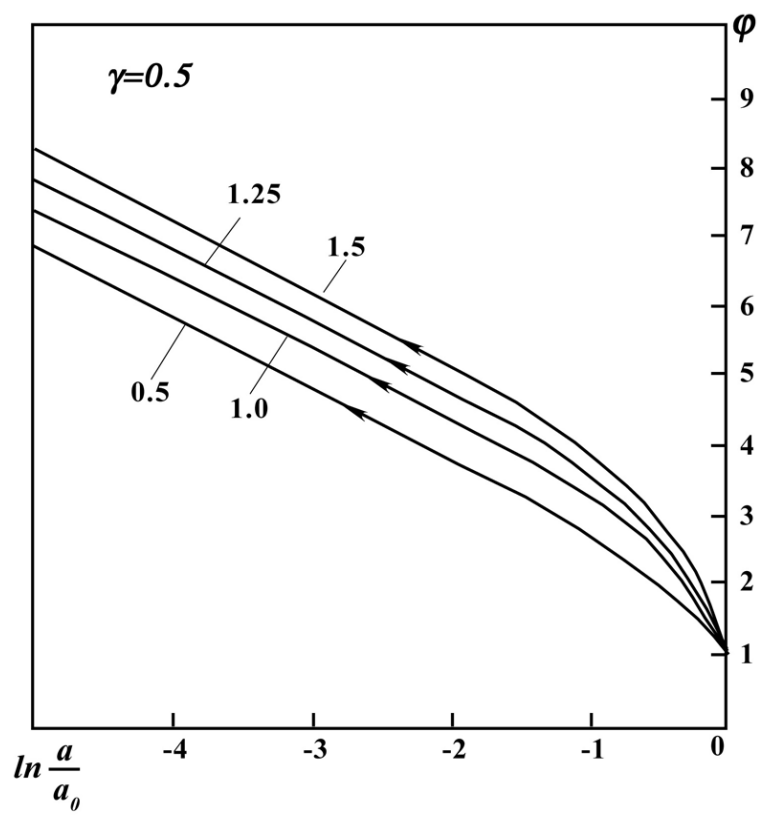

(b)

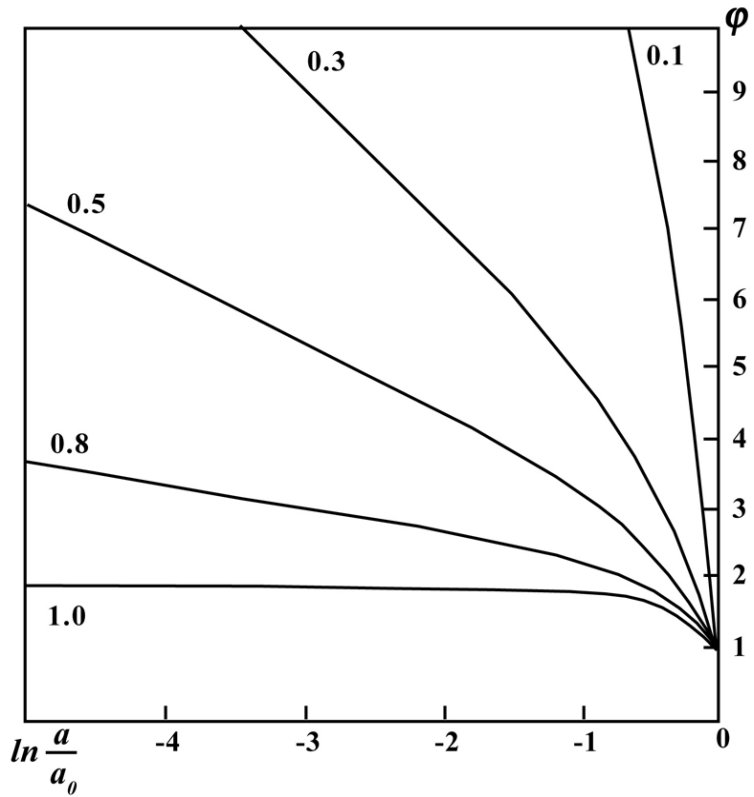

Fig. 3. The stream-lines of the nonlinear fractional oscillator (64) in the phase plane.

\section{Conclusion}

An engineering analytical approach is proposed for the approximate analysis of dynamic behavior of linear and nonlinear fractional oscillators. For this purpose, in this paper the fractional derivative is determined as a fractional power of the operator of conventional time-derivative, what allows one to take no account for the drift of the equilibrium position of the oscillator.

The comparison of the obtained results is carried out for free damped vibrations of the oscillators with the operator $(d / d t)^{\gamma}$ in damping description for the cases of small and finite viscosity, as well as for the oscillator with the fractional operator in inertial force description. It has been shown that in first two cases it is observed an approximate 
proportionality in the behavior of the damping coefficients and the frequencies of vibration as functions of the relaxation (retardation) time. As for the oscillator possessing 'fractional inertia', then it has been demonstrated that the formal replacement of the second time-derivative of the displacement by a fractional one of order less than two in the equation of motion of the mechanical oscillator is unjustified from the point of view of engineering practice.

It has been shown that, depending on the order of low-level of the fractional derivative, the solutions obtained for free damped vibrations of the nonlinear mechanical oscillator by the method of multiple time scales differ from each other by the character of nonlinearity: the interrelationship of the fractional parameter and nonlinearity manifests itself in full measure when orders of the smallness of the fractional derivative and of the cubic nonlinearity entering in the oscillator's constitutive equation coincide. In the other case, a correlation between them is weak.

The influence of the initial conditions and the fractional parameter on the behavior of the stream-lines of the phase fluid has been analysed. It has been shown that the steady-state motion of the phase fluid corresponds to the steady-state vibrations of the nonlinear oscillator.

The engineering approach implemented in this paper to the analysis of free vibrations of mechanical oscillators, constitutive equations of which involve fractional derivatives, can be easily extended for investigating forced vibrations of such oscillators.

\section{Acknowledgements}

The research described in this publication has been made possible in part by the joint Grant from the Russian Foundation for Basic Research No.07-01-92002-HHC-a and the National Science Council of Taiwan No.96WFA2500005.

This paper has been completed during the authors' stay at Rice University, TX as Visiting Professors. The second author wishes to thank the Fulbright Foundation for the opportunity to do research at Rice University through the Fulbright Fellowship.

\section{Appendix A}

Formula (10) can be obtained from the Mellin-Fourier formula for the function $D_{0+}^{\gamma} e^{i \omega t}$. Really,

$$
D_{0+}^{\gamma} e^{i \omega t}=\frac{1}{2 \pi i} \int_{c-i \infty}^{c+i \infty} \frac{p^{\gamma} e^{p t} d p}{p-i \omega} .
$$

To calculate the integral in the right-hand side of Eq. (A1) we use the contour of integration $L$ shown in Fig. 4 . Applying the theorem of the theory of residues for the integral over the contour $L$, we have

$$
\frac{1}{2 \pi i} \int_{L} \frac{p^{\gamma} e^{p t} d p}{p-i \omega}=(i \omega)^{\gamma} e^{i \omega t}
$$

Writing the contour integral in terms of the sum of integrals along the vertical segment of the straight line, along the arcs of the circumferences with radii $C_{R}$ and $C_{\rho}$, and along the branches of the cut of the negative real semi-axis, then tending $R$ to $\infty$ and $\rho$ to 0 , and considering Jordan lemma, we arrive at the relationship (10).

Changing in Eq. (10) $i \omega$ by $-i \omega$ yields

$$
D_{0+}^{\gamma} e^{-i \omega t}=(-i \omega)^{\gamma} e^{-i \omega t}+\frac{\sin \pi \gamma}{\pi} \int_{0}^{\infty} \frac{u^{\gamma} e^{-u t} d u}{u-i \omega} .
$$

Adding Eqs (10) and (A3), we obtain

$$
D_{0+}^{\gamma}(\cos \omega t)=\omega^{\gamma} \cos \left(\omega t+\frac{\pi}{2} \gamma\right)+\frac{\sin \pi \gamma}{\pi} \int_{0}^{\infty} \frac{u^{1+\gamma} e^{-u t} d u}{u^{2}+\omega^{2}}
$$

while subtracting Eq. (A3) from Eq. (10) we have 


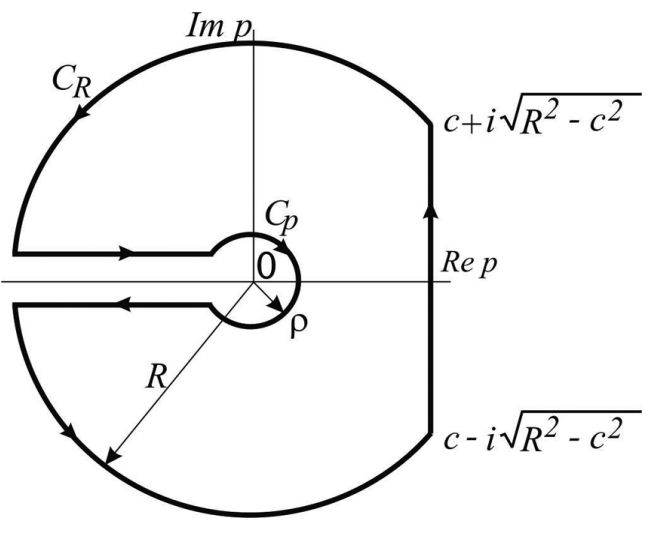

Fig. 4. The contour of integration.

$$
D_{0+}^{\gamma}(\sin \omega t)=\omega^{\gamma} \sin \left(\omega t+\frac{\pi}{2} \gamma\right)-\frac{\sin \pi \gamma}{\pi} \omega \int_{0}^{\infty} \frac{u^{\gamma} e^{-u t} d u}{u^{2}+\omega^{2}}
$$

When $\gamma=1$, these formulas go over into the corresponding formulas of conventional differentiation.

The derived Eqs (10) and (A3)-(A5) are more preferred in engineering applications than those presented in Table 9.1 of [34] or in tables for semiderivatives in Chapter 7 [55], since allow one to estimate the accuracy of approximate solutions like those constructed in the given paper.

Reference to Eqs (10) and (A3)-(A5) shows that it is possible to ignore the improper integrals in the following cases: 1) if $\gamma$ differs a little from unit, 2) when the magnitude of the fractional parameter $\gamma$ is rather small, 3) for rather large frequencies $\omega$. The second terms in Eqs (10) and (A3)-(A5) could be also neglected from some instants of time after the beginning of vibratory motion.

It should be emphasized that in some engineering problems a combination of the conditions mentioned above can occur at a time, what allows one to use Eq. (9).

Taking no account of the improper integrals is tantamount to replacing the derivative $D_{0+}^{\gamma}$ with the derivative $D_{+}^{\gamma}$ in Eqs (10) and (A3)-(A5). Such a substitution has been used in the given paper for the analysis of linear and nonlinear fractional oscillators.

Note that the similar formulas were first derived by Caputo [66] using the Caputo fractional derivative representation. The estimates of improper integrals useful for engineering applications were suggested in [66] as well.

\section{References}

[1] Yu.A. Rossikhin and M.V. Shitikova, Application of fractional calculus to dynamic problems of linear and nonlinear hereditary mechanics of solids, Applied Mechanics Reviews 50 (1997), 15-67.

[2] P. Arena, R. Caponetto, L. Fortuna and D. Porto, Nonlinear Noninteger Order Circuits and Systems An Introduction, World Scientific, Singapore-New Jersey-London-Hong Kong 2000.

[3] L. Debnath, Recent applications of fractional calculus to science and engineering, Int J M M S 54 (2003), 3413-3442.

[4] R.L. Magin, Fractional calculus in bioengineering, Critical Reviews in Biomedical Engineering 32 (2004), 1-104.

[5] G.E. Draganescu, Application of a variational iteration method to linear and nonlinear viscoelastic models with fractional derivatives, Journal of Mathematical Physics 47 (2006), 082902-1-9.

[6] M. Xu and W. Tan, Intermediate processes and critical phenomena: Theory, method and progress of fractional operators and their applications to modern mechanics, Science in China Ser G: Physics, Mechanics and Astronomy 49 (2006), 257-272.

[7] M.S. Tavazoei and M. Haeri, Regular oscillations or chaos in a fractional order system with any effective dimension, Nonlinear Dynamics (2008) DOI 10.1007/s11071-007-9323-1.

[8] B.N.N. Achar, J.W. Hanneken, T. Enck and T. Clarke, Dynamics of the fractional oscillator, Physica A 297 (2001), 361-367.

[9] G.M. Zaslavsky, A.A. Stanislavsky and M. Edelman, Chaotic and pseudochaotic attractors of perturbed fractional oscillator, Chaos 16 (2006), 013102-1-6.

[10] R.S. Barbosa, J.A.T. Machado, B.M. Vinagre and A.J. Calderon, Analysis of the Van der Pol oscillator containing derivatives of fractional order, Journal of Vibration and Control 13 (2007), 1291-1301.

[11] J.H. Chen and W.C. Chen, Chaotic dynamics of the fractionally damped van der Pol equation, Chaos, Solitons and Fractals 35 (2008), $168-198$. 
[12] V.E. Tarasov and G.M. Zaslavsky, Dynamics with low-level fractionality, Physica A 368 (2006), 399-415.

[13] Yu.A. Rossikhin and M.V. Shitikova, Application of fractional derivatives to the analysis of damped vibrations of viscoelastic single mass systems, Acta Mechanica 120 (1997), 109-125.

[14] Yu.A. Rossikhin and M.V. Shitikova, Application of fractional operators to the analysis of damped vibrations of viscoelastic single-mass systems, Journal of Sound and Vibration 199 (1997), 567-586.

[15] Yu.A. Rossikhin and M.V. Shitikova, Application of fractional derivatives for the analysis of nonlinear damped vibrations of suspension bridges, Proceedings of 1997 International Symposium on Nonlinear Theory and its Applications 1, Honolulu, USA, Nov 29 - Dec 2, 1997, 541-544.

[16] Yu.A. Rossikhin and M.V. Shitikova, Application of fractional calculus for analysis of nonlinear damped vibrations of suspension bridges, ASCE Journal of Engineering Mechanics 124 (1998), 1029-1036

[17] J. Padovan and J.T. Sawicki, Nonlinear vibration of fractionally damped systems, Nonlinear Dynamics 16 (1998), 321-336.

[18] Yu.A. Rossikhin and M.V. Shitikova, Analysis of nonlinear vibrations of a two-degree-of-freedom mechanical system with damping modelled by a fractional derivative, Journal of Engineering Mathematics 37 (2000), 343-362.

[19] M. Seredynska and A. Hanyga, Nonlinear differential equations with fractional damping with applications to the 1dof and 2dof pendulum, Acta Mechanica 176 (2005), 169-183.

[20] Yu.A. Rossikhin and M.V. Shitikova, Analysis of free non-linear vibrations of a viscoelastic plate under the conditions of different internal resonances, International Journal of Non-Linear Mechanics 41 (2006), 313-325.

[21] J.H. He, Approximate analytical solution for seepage flow with fractional derivatives in porous media, Computer Methods in Applied Mechanics and Engineering 167 (1998), 57-68.

[22] L.J. Sheu, H.K. Chen, J.H. Chen and L.M. Tam, Chaotic dynamics of the fractionally damped Duffing equation, Chaos, Solitons, Fractals 32 (2007), 1459-1468.

[23] M. Seredynska and A. Hanyga, Nonlinear Hamiltonian equations with fractional damping, Journal of Mathematical Physics 41 (2000), 2135-2156.

[24] X. Gao and J. Yu, Chaos in the fractional order periodically forced complex Duffing's oscillators, Chaos, Solitons and Fractals 24 (2005), 1097-1104.

[25] S.J. Singh and A. Chatterjee, Galerkin projections and finite elements for fractional order derivatives, Nonlinear Dynamics 45 (2006), 183-206.

[26] P. Wahi and A. Chatterjee, Averaging oscillators with small fractional damping and delayed terms, Nonlinear Dynamics 38 (2004), 3-22.

[27] R. Deng, P. Davies and A.K. Bajaj, Flexible polyurethan foam modelling and identification of viscoelastic parameters for automotive seating applications, Journal of Sound and Vibration 262 (2003), 391-417.

[28] R.E. Mickens, K.O. Oyedeji and S.A. Rucker, Analysis of the simple harmonic oscillator with fractional damping, Journal of Sound and Vibration 268 (2003), 839-842.

[29] R.E. Mickens, Fractional van der Pol equations, Journal of Sound and Vibration 259 (2003), 457-460.

[30] J.I. Ramos, Piecewise-linearized methods for oscillators with fractional power nonlinearities, Journal of Sound and Vibration 300 (2007), $502-521$.

[31] G. Terenzi, Dynamics of SDOF systems with nonlinear viscous damping, ASCE Journal of Engineering Mechanics 125 (1999), $956-963$.

[32] M. Borowiec, G. Litak and A. Syta, Vibration of the Duffing oscillator: Effect of fractional damping, Shock and Vibration 14 (2007), 29-36.

[33] G. Litak, M. Borowiec and A. Syta, Vibration of generalized double well oscillators, ZAMM 87 (2007), 590-602.

[34] S.G. Samko, A.A. Kilbas and O.I. Marichev, Fractional Integrals and Derivatives. Theory and Applications (in Russian), Nauka i Tekhnika, Minsk 1988 (Engl. transl. by Gordon and Breach Science Publ., Amsterdam 1993).

[35] A.M. Abdel-Ghaffar and R.H. Scanlan, Ambient vibration studies of Golden Gate Bridge. I: Suspended structure, ASCE Journal of Engineering Mechanics 111 (1985), 463-482.

[36] Yu.A. Rossikhin and M.V. Shitikova, Nonlinear free damped vibrations of suspension bridges with uncertain fractional damping, JESA 42 (2008).

[37] M. Giovagnoni and G. Berti, A fractional derivative model for single-link mechanism vibration, Meccanica 27 (1992), 131-138.

[38] A. Schmidt and L. Gaul, On the numerical evaluation of fractional derivatives in multi-degree-of-freedom systems, Signal Processing 86 (2006), 2592-2601.

[39] J.A. Cooke and R.F. Keltie, Determination of the impulse response of a viscoelastic beam using a fractional derivative constitutive model, ASME Des. Eng. Div. Publ. DE-5 (1987), 137-141.

[40] I. Schäfer, Beschreibung der Dämpfung in Stäben mittels fraktionaler Zeitableitungen (in German), ZAMM 80 (2000), 356-360.

[41] I. Schäfer and H.-J. Seifert, Description of the impulse response in rods by fractional derivatives, ZAMM 82 (2002), $423-427$.

[42] C.G. Koh and J.M. Kelly, Application of fractional derivatives to seismic analysis of base-isolated models, Earthquake Engineering and Structural Dynamics 19 (1990), 229-241.

[43] N. Makris and M.C. Constantinou, Fractional-derivative Maxwell model for viscous dampers, ASCE Journal of Structural Engineering 117 (1991), 2708-2724.

[44] J.S. Hwang and S.W. Ku, Analytical modeling of high damping rubber bearings, ASCE Journal of Structural Engineering 123 (1997), 1029-1036.

[45] A. Aprile, J.A. Inaudi and J.M. Kelly, Evolutionary model of viscoelastic dampers for structural applications, ASCE Journal of Engineering Mechanics 123 (1997), 551-560.

[46] J.A. Munshi, Effect of viscoelastic dampers on hysteretic response of reinforced concrete elements, Engineering Structures 19 (1997), 921-935.

[47] J.S. Hwang and T.Y. Hsu, A fractional derivative model to include effect of ambient temperature on HDR bearings, Engineering Structures 23 (2001), 484-490. 
[48] V. Gusella and G. Terenzi, Time-dependent analysis of structures with dampers modelled by fractional derivatives, Advances in Earthquake Engineering 9 (2001), 251-260.

[49] M. Sjöberg and L. Kari, Nonlinear isolator dynamics at finite deformations: An effective hyperelastic, fractional derivative, generalized friction model, Nonlinear Dynamics 33 (2003), 323-336.

[50] Yu.A. Rossikhin and M.V. Shitikova, Analysis of dynamic behaviour of viscoelastic rods whose rheological models contain fractional derivatives of two different orders, ZAMM 81 (2001), 363-376.

[51] Yu.A. Rossikhin and M.V. Shitikova, Analysis of the viscoelastic rod dynamics via models involving fractional derivatives or operators of two different orders, The Shock and Vibration Digest 36 (2004), 3-26.

[52] O.P. Agrawal, Analytical solution for stochastic response of a fractionally damped beam, ASME Journal of Vibration and Acoustics $\mathbf{1 2 6}$ (2004), 561-566.

[53] I. Schäfer and S. Kempfle, Impulse responses of fractional damped systems, Nonlinear Dynamics 38 (2004), 61-68.

[54] A.H. Nayfeh, Perturbation Methods, Wiley, New York 1973.

[55] K.B. Oldham and J. Spanier, The Fractional Calculus, Academic Press, New York 1974.

[56] L.E. Suarez and A. Shokooh, Response of systems with damping materials modeled using fractional calculus, Applied Mechanics Reviews 48 (1995), Part 2, S118-S126.

[57] S. Sakakibara, Properties of vibration with fractional derivative damping of order 1/2, JSME International Journal Series C, 40 (1997), 393-399.

[58] N. Shimizu and M. Iijima, Impulse reponses of viscoelastic oscillator (in Japanese), Transactions of the Japanese Society of Mechanical Engineeres C 64 (1997), 2903-2907.

[59] W. Zhang and N. Shimizu, Damping properties of the viscoelastic material described by fractional Kelvin-Voigt model, JSME International Journal Series C, 42 (1999), 1-9.

[60] V.M. Zelenev, S.I. Meshkov and Yu.A. Rossikhin, Damped vibrations of hereditary-elastic systems with weakly singular kernels, Applied Mechanics and Technical Physics 11 (1970), 290-293.

[61] V.S. Ekel'chik and V.M. Ryabov, Free vibrations of a hereditary-elastic polymer rod in the transition from the glassy state to the highly elastic state, Mechanics of Composite Materials 23 (1987), 376-381.

[62] B.N.N. Achar, J.W. Hanneken and T. Clarke, Response characteristics of a fractional oscillator, Physica A 309 (2002), 275-288.

[63] B.N.N. Achar, J.W. Hanneken and T. Clarke, Damping characteristics of a fractional oscillator, Physica A 339 (2004), $311-319$.

[64] A. Tofighi, The intrinsic damping of the fractional oscillator, Physica A 329 (2003), 29-34.

[65] Yu.A. Rossikhin, M.V. Shitikova and T.A. Shcheglova, Nonlinear dynamics of a fractionally damped oscillator, in Proceedings of the 10th Pan American Congress on Applied Mechanics, Jan 7-11, 2008, Cancun, Mexico, 223-226.

[66] M. Caputo, Vibrations of an infinite viscoelastic layer with a dissipative memory, Journal of the Acoustical Society of America 56 (1974), 897-904. 

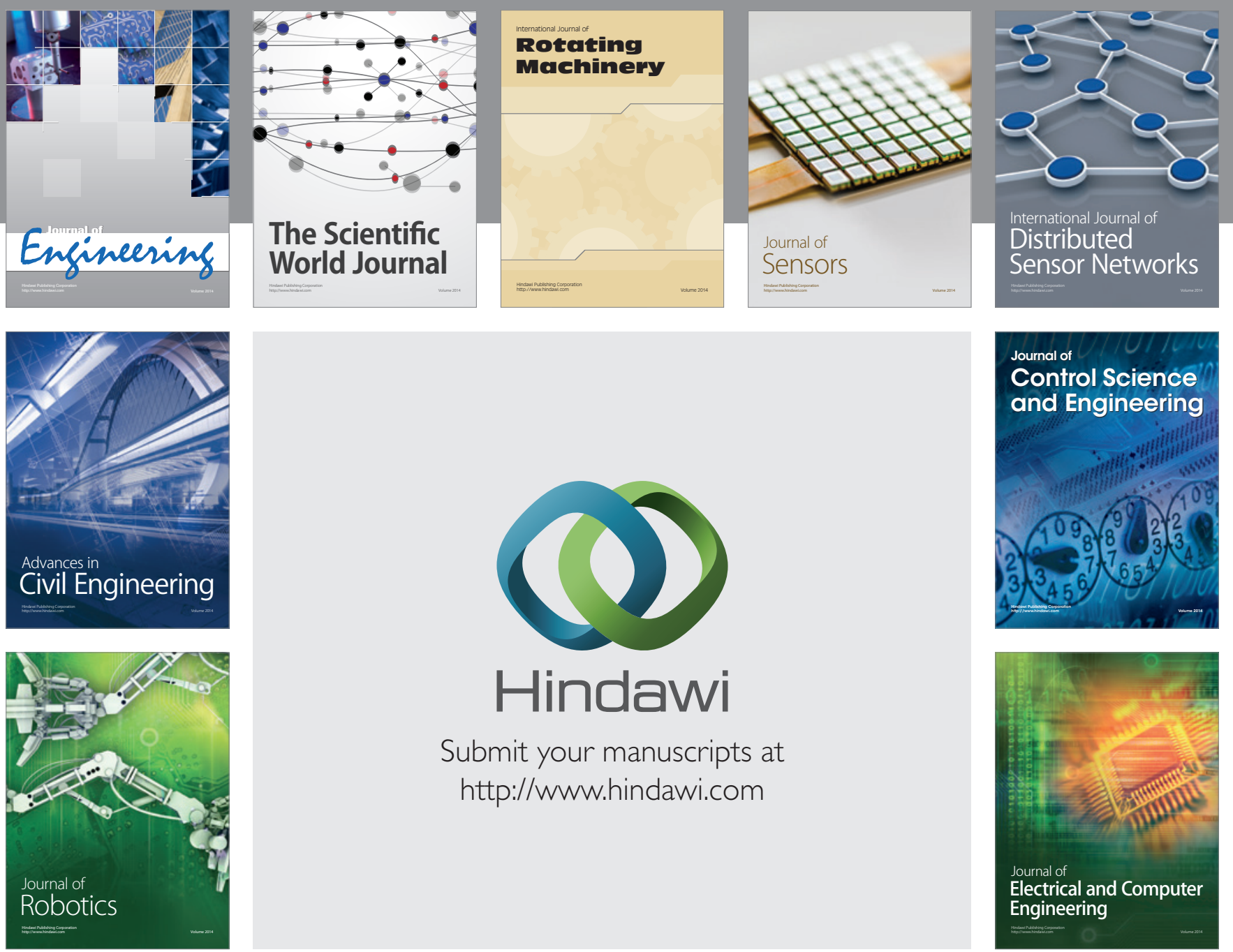

Submit your manuscripts at

http://www.hindawi.com
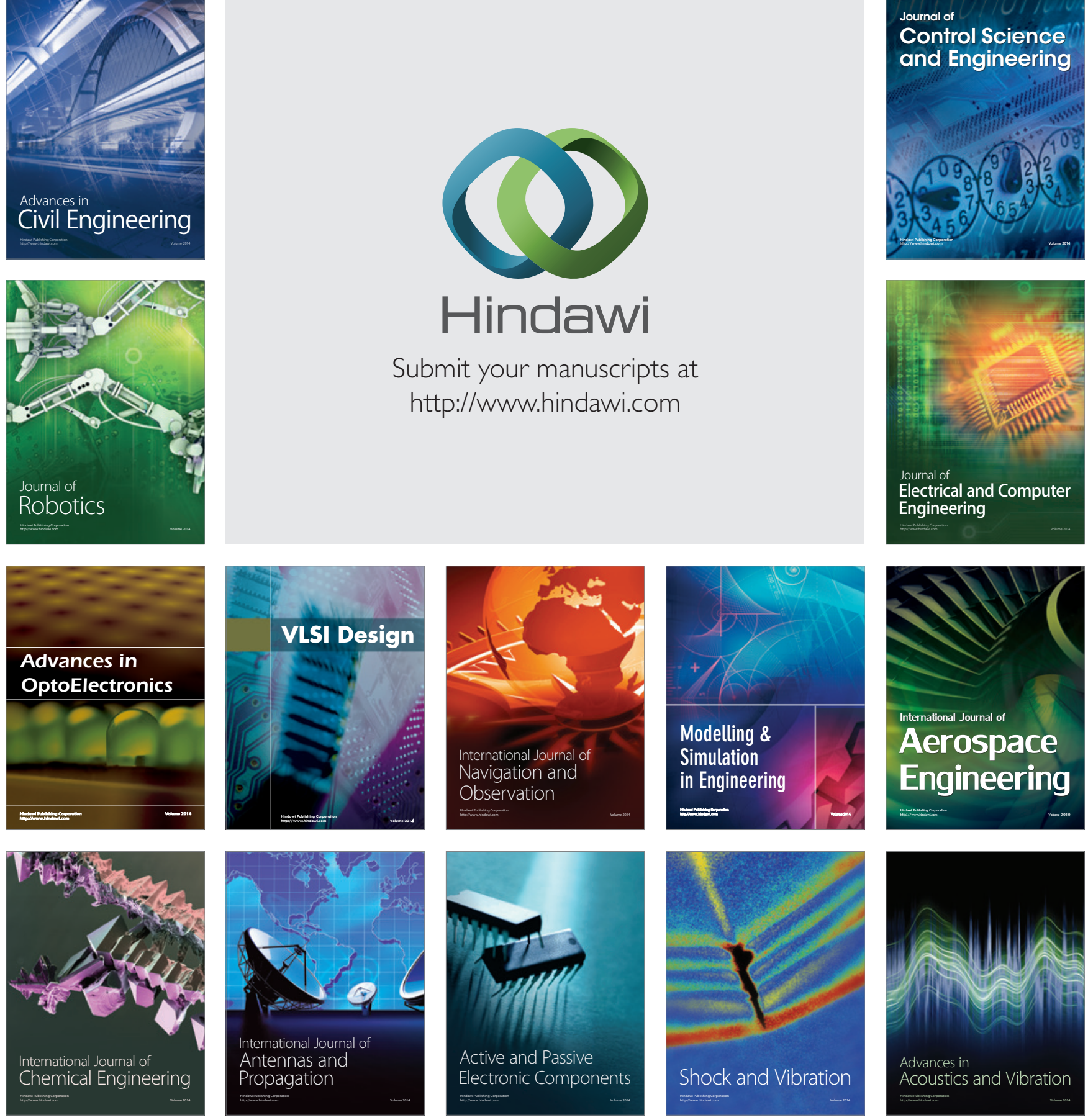